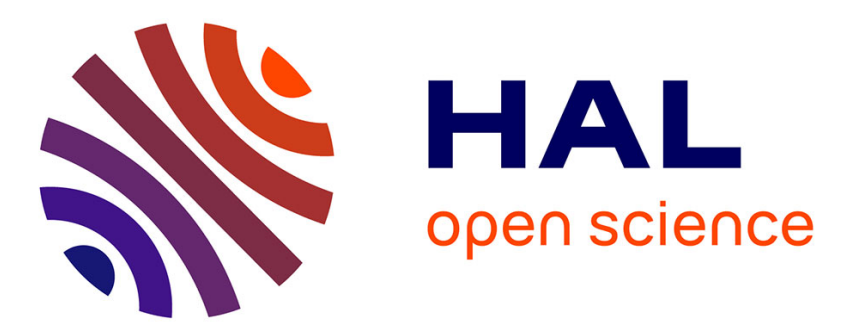

\title{
Evaporation of a sodium chloride solution from a saturated porous medium with efflorescence formation
}

Stéphanie Veran-Tissoires, Marc Prat

\section{To cite this version:}

Stéphanie Veran-Tissoires, Marc Prat. Evaporation of a sodium chloride solution from a saturated porous medium with efflorescence formation. Journal of Fluid Mechanics, 2014, vol. 749, pp.701-749. 10.1017/jfm.2014.247 . hal-01002166

\section{HAL Id: hal-01002166 https://hal.science/hal-01002166}

Submitted on 5 Jun 2014

HAL is a multi-disciplinary open access archive for the deposit and dissemination of scientific research documents, whether they are published or not. The documents may come from teaching and research institutions in France or abroad, or from public or private research centers.
L'archive ouverte pluridisciplinaire HAL, est destinée au dépôt et à la diffusion de documents scientifiques de niveau recherche, publiés ou non, émanant des établissements d'enseignement et de recherche français ou étrangers, des laboratoires publics ou privés. 


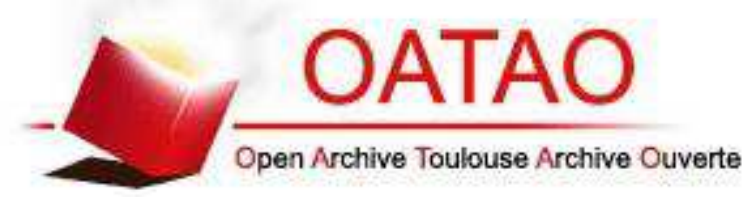

\section{Open Archive TOULOUSE Archive Ouverte (OATAO)}

OATAO is an open access repository that collects the work of Toulouse researchers and makes it freely available over the web where possible.

This is an author-deposited version published in : http://oatao.univ-toulouse.fr/ Eprints ID : 11732

To link to this article :

DOI: $10.1017 / \mathrm{jfm} .2014 .247$

URL : http://dx.doi.org/10.1017/jfm.2014.247

\section{To cite this version :}

Veran-Tissoires, Stéphanie and Prat, Marc Evaporation of a sodium chloride solution from a saturated porous medium with efflorescence formation. (2014) Journal of Fluid Mechanics, vol. 749 . pp. 701-749.

Any correspondance concerning this service should be sent to the repository administrator: staff-oatao@ listes-diff.inp-toulouse.fr 


\title{
Evaporation of a sodium chloride solution from a saturated porous medium with efflorescence formation
}

\author{
Stéphanie Veran-Tissoires and Marc Prat $\dagger$ \\ Université de Toulouse; INPT, UPS; IMFT, Avenue Camille Soula, 31400 Toulouse, France CNRS; \\ IMFT, 31400 Toulouse, France
}

\begin{abstract}
Precipitation of sodium chloride driven by evaporation at the surface of a porous medium is studied from a combination of experiments, continuum simulations, pore network simulations and a simple efflorescence growth model on a lattice. The distribution of ions concentration maxima at the porous medium surface, which are seen as the incipient precipitation spots, is shown to be strongly dependent on the factors affecting the velocity field within the porous medium owing to the significance of advection on ion transport. These factors include the evaporation flux distribution at the surface at Darcy's scale as well as the scale of surface menisci and the internal disorder of the porous medium, which induce spatial fluctuations in the velocity field. The randomness of the velocity field within the porous medium and at its surface explains the discrete nature of incipient precipitation spots at the surface of a porous medium. Experiments varying the mean size of the beads forming the porous medium lead to the identification of two main types of efflorescence, referred to as crusty and patchy, and the impact of these two types on evaporation is completely different. The crusty efflorescence severely reduces the evaporation rate whereas the patchy efflorescence can enhance the evaporation rate compared with pure water. The crusty-patchy transition is analysed from a simple growth model on a lattice taking into account the porous nature of efflorescence structures.
\end{abstract}

Key words: low-Reynolds-number flows, porous media

\section{Introduction}

Salt crystallization in porous media or at the surface of a porous medium is a phenomenon of interest in relation with several important applications, such as soil physics, underground storage of $\mathrm{CO}_{2}$, civil engineering and the protection of our cultural heritage, to name only a few. The soil-physics-related issues concern the impact of salt on evaporation of water from soils and the associated impacts on many aspects of water management, global water cycle, soil and groundwater salinization, agriculture (e.g. Nachshon et al. 2011, and references therein). As discussed in Peysson et al. (2011), the evaporation process occurring during the injection of $\mathrm{CO}_{2}$ in a saline aquifer can induce the dissolved salt precipitation in the porous

$\dagger$ Email address for correspondence: mprat@imft.fr 
structure. The result can be the filling of the pores by the crystallized salts inducing in turn a severe decrease in the permeability. Permeability alteration by salting out is thus a serious risk of injectivity decline in the context of $\mathrm{CO}_{2}$ geological storage in saline aquifers where high levels of gas injection have to be maintained over decades. The applications having motivated the largest number of studies are related to the salt weathering issues Goudie \& Viles (1997). As discussed for example by Scherer (2004), Coussy (2006) and Schiro, Ruiz-Agudo \& Rodriguez-Navarro (2012), the crystallization process in the pores generates pressure against the pore walls that might eventually result in damage to the host material. The induced internal stresses can be sufficient to completely destroy a stone subjected to evaporation (e.g. Rodriguez-Navarro \& Doehne 1999). The salt crystallization process is therefore a major cause of deterioration of sculpture and monuments made of stone and masonry. For example, it is generally admitted that salt crystallization is one of the greatest threats to monuments in the Mediterranean basin (Theoulakis \& Moropoulou 1999).

In spite of the significance of these various applications, the understanding of the interplay between the salt crystallization and evaporation processes is not very advanced and many aspects need to be clarified. As mentioned before, the salt crystallization process in porous materials has motivated many works but only very few of them focus on the impact of crystallization on the evaporation process or on the detailed understanding of the factors controlling the localization of crystallized salt structures within or at the surface of a porous medium subjected to evaporation. This is however a crucial aspect before attempting any poromechanical analysis. The crystallized salt structures forming inside a porous medium are referred to as subflorescence whereas the crystallized structures forming at the surface are referred to as efflorescence.

Two main reference situations can be distinguished in this context (Scherer 2004): drying and evaporation-wicking. In drying (e.g. Huinink, Pel \& Michels 2002; Sghaier, Prat \& Ben Nasrallah 2007; Guglielmini et al. 2008; Gupta et al. 2012; Eloukabi et al. 2013; Hidri et al. 2013; Norouzi Rad, Shokri \& Sahimi 2013), the limiting surfaces of the porous sample are in contact with impervious walls or exposed to evaporation. In the evaporation-wicking situation (e.g. Puyate \& Lawrence 1998; Puyate et al. 1998; Puyate \& Lawrence 1999; Veran-Tissoires, Marcoux \& Prat 2012a,b), the difference with drying is that the sample is in contact at its bottom with an aqueous solution. The liquid is drawn into the pores by capillary suction. In contrast with drying, there is a permanent supply of solution and therefore a steady-state can possibly be reached when the flow rate of liquid sucked into the medium by capillary action exactly balances the evaporation rate. The medium can be fully saturated by the solution when the capillary action is sufficiently strong or an internal evaporation front can exist when the capillary action is insufficient to maintain the medium fully saturated. This leads to eventually identify three main generic situations: drying, (e.g. Eloukabi et al. 2013; Hidri et al. 2013), evaporation-wicking with the medium fully saturated (e.g. Veran-Tissoires et al. 2012a,b) and evaporation-wicking with an internal evaporation front (e.g. Noiriel et al. 2010). These three related but different situations correspond to real situations (e.g. Scherer 2004). For example, the fully saturated evaporation-wicking case can correspond to the section of a wall near the ground, whereas an internal evaporation front can exist at higher elevations along the wall. The drying case can apply to stones at still higher elevations, where the stones cease to be connected to the solution in the ground at one time or another.

In this paper, we focus on the evaporation-wicking situation, where the porous medium remains fully saturated all along the evaporation process. The situation we 
focus on is sketched in figure 1. A porous medium is exposed to evaporation at its top surface and is in contact with a sodium chloride aqueous solution at its bottom. This is the same situation as that considered by Veran-Tissoires et al. $(2012 a, b)$. The case of a homogeneous porous medium was considered in Veran-Tissoires et al. (2012a), whereas the case of a heterogeneous porous medium made of a column of a fine porous medium set in the middle of a coarse porous medium was considered in Veran-Tissoires et al. (2012b). Both papers contribute to elucidate the factors controlling the localization of efflorescence structures at the evaporative surface of porous medium. These factors are the distribution of evaporation flux at the surface and the local (homogeneous porous medium) and large-scale (heterogeneous porous medium) disorder of the porous medium. These factors affect the structure of the velocity field within the porous medium. Under usual evaporation conditions, the transport of the ions by this heterogeneous and random velocity field has a dominant effect on the ion concentration at the surface and therefore on the localization of concentration maxima, which correspond to the places where precipitation first occurs. Then the fact that the efflorescence structures continue to grow as well-individualized structures was explained by the progressive reorganization of the velocity field in the underlying porous media. This reorganization is due to the screening of evaporation flux in the regions of surface located between the efflorescence structures and the preferential sucking effect associated with the porous nature of the salt structures as the salt structures grow. These results were presented in two short letters. However, two important aspects were not discussed at all. First, the efflorescence does not always take the form of a series of well-individualized growing structures. The efflorescence can form a compact crust, generally blocking or severely limiting the evaporation. Second, the disordered nature of the porous induces local fluctuations in the evaporation flux at the surface. These fluctuations were not taken into account properly in the analysis. Also, the effect of supersaturation was overlooked.

In addition to these important points, the objective is to present additional elements not reported in Veran-Tissoires et al. $(2012 a, b)$. In particular, we emphasize the dynamic aspect of efflorescence structure growth. The combination of crystallization and salt redissolution processes at the surface of the porous medium leads to a sort of efflorescence dance when the film of the successive images of the surface during the evaporation-crystallization process at the surface is viewed at an accelerated speed.

The paper is organized as follows. The experimental set-up is presented in $\S 2$. Section 3 is devoted to a numerical study of the distribution of evaporation flux at the surface of the porous medium from both a continuum and a discrete approach. The factors affecting the distribution of ion concentration local maxima at the porous medium surface and the time of first precipitation are studied in $\S 4$ from a combination of experiments, continuum simulations and pore network simulations. Section 5 presents some dynamic aspects of efflorescence growth, indicating that efflorescence growth should be considered, in fact, as a dissolution-crystallization process. The effect of porous medium pore size is explored in $\S 6$, which leads to the identification two types of efflorescence, referred to as crusty and patchy. The crusty efflorescence severely reduces the evaporation rate whereas the patchy efflorescence can increase the evaporation rate. The crusty-patchy transition is analysed from a simple growth model of efflorescence on a lattice. A summary of the work and some possible future works are presented in the conclusion.

\section{Experimental set-up}

The experimental set-up is sketched in figure 2. A pack of glass beads of mean size $d_{b}$ in a $50 \mathrm{~mm}$ long hollow cylinder of inner radius $r_{w}=19 \mathrm{~mm}$ forms a porous 


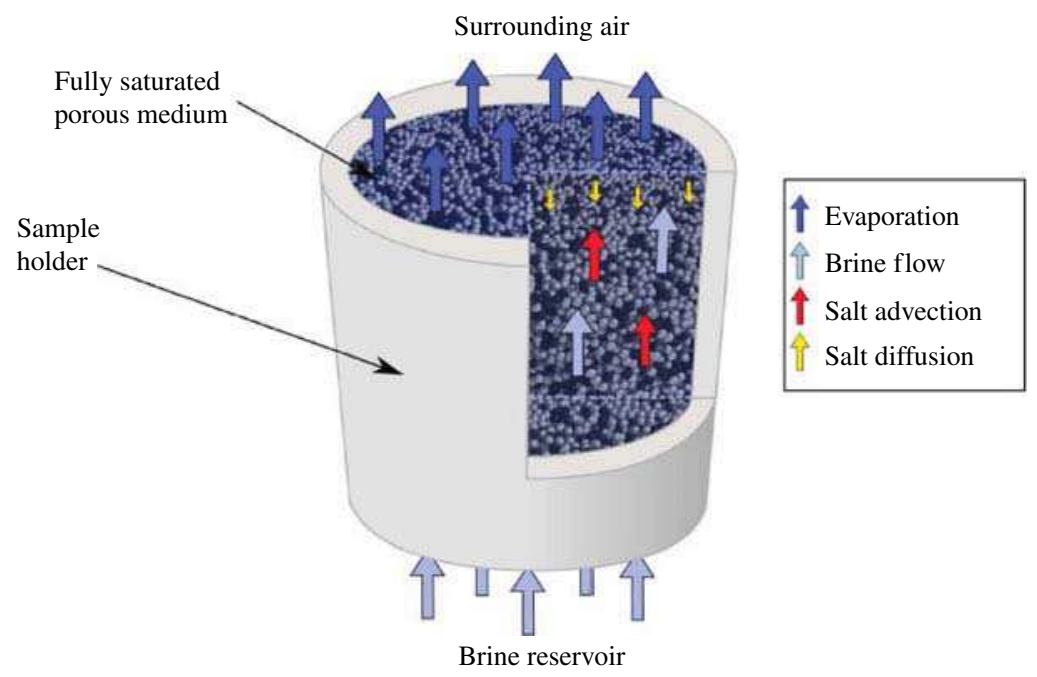

FIGURE 1. (Colour online) Sketch of the evaporation-wicking situation considered in the article. A porous medium is in contact with a sodium chloride aqueous solution at its bottom and exposed to evaporation at the top surface. The medium remains fully saturated owing to capillary action at the top. The evaporation process combined with the capillary action induces a flow all along the porous medium balancing at the top the evaporation.

medium. The packing of height $L$ is in contact at its bottom with a sodium chloride almost saturated aqueous solution (the initial salt mass fraction is $C_{0}=25 \%$; the saturation mass fraction is $C_{s a t}=26.4 \%$ ). The liquid level $h$ in the reservoir is at any time such that the porous medium remains fully saturated owing to capillary suction. The system is set in a cylindrical enclosure of controlled temperature $\left(T \approx 22^{\circ} \mathrm{C}\right)$ and relative humidity (thanks to a $\mathrm{LiBr}$ saline solution, relative humidity $R H \approx 7 \%$ ). The wick being fully saturated with the aqueous solution (see below), evaporation takes place at the top surface of the wick. Note that the cylinder containing the porous medium is open at the top. Thus, the porous medium surface is in contact with the dryer air in the enclosure.

The evaporation rate at the wick surface is varied by changing the distance $\delta$ between the wick surface and the hollow cylinder entrance (this will be clarified in $\S 4$ ). In a first set of experiments with beads $1 \mathrm{~mm}$ in diameter, three different distances $\delta$ between the packing surface and the rim of the hollow cylinder, namely 2.5, 7.5 and $15 \mathrm{~mm}$ are considered. In a second set of experiments focusing on the effect of bead diameter on the salt precipitation/evaporation process $\delta$ will be set constant at $15 \mathrm{~mm}$. Four ranges of bead diameters are then used: $(5-50 \mu \mathrm{m})$, (50-62 $\mu \mathrm{m}),(100-120 \mu \mathrm{m})$ and $(1-1.125 \mathrm{~mm})$. The two series of experiments also differ by the position of the free surface in the aqueous solution reservoir, i.e. the height $h$ or equivalently the vertical distance $L_{s}$ between the free surface in the reservoir and the surface of the porous medium (see figure 2). In the first set of experiments, which focuses on the effect of $\delta$, the distance $L_{s}$ was not set carefully. This distance was of the order of $10 \mathrm{~mm}$ or less and the idea was just to make sure that the medium was fully saturated all along the experiment. In the second set of experiments on the impact of bead diameter, the distance was set carefully at the beginning of each experiment with $L_{s}=22 \mathrm{~mm}$, thus greater than in the first series of experiments. 


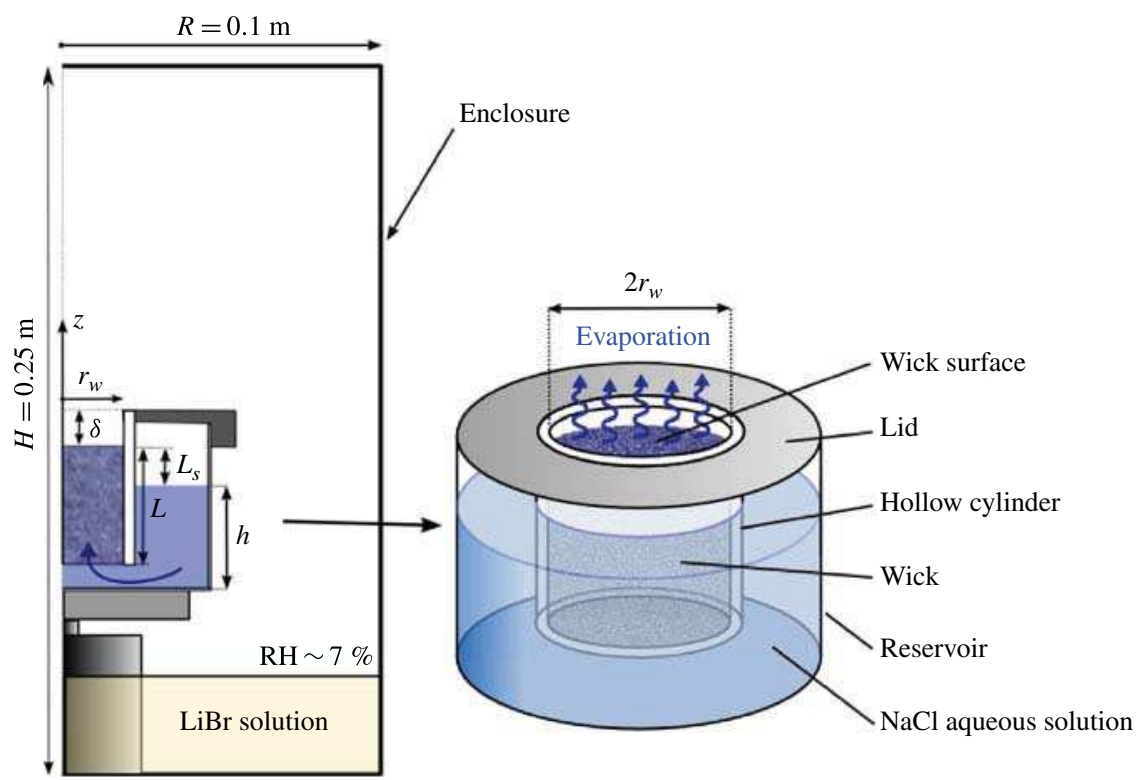

FiguRE 2. (Colour online) Sketch of experimental set-up. Note the enclosure which corresponds to the computational domain for the transport of the vapour as described in the text.

The medium remains saturated during the experiments. A simple computation helps estimate the level $h$ of the solution that should be imposed in the reservoir containing the solution. As reported in Masoodi, Pillai \& Varanasi (2007) the maximum suction in a packed bed of monodisperse beads can be estimated by $P_{c}=6(1-\varepsilon) \gamma \cos \theta / \varepsilon d_{b}$ where $\gamma$ is the surface tension, $\theta$ the contact angle, $\varepsilon$ the porosity and $d_{b}$ the bead diameter. The surface tension increases with the salt concentration but the product $\gamma \cos \theta$ does not vary significantly (Sghaier, Prat \& Ben Nasrallah 2006). As a result, one can safely take the value for pure water. Assuming, for example, $\theta=45^{\circ}$, which is a conservative upper bound according to the measurements of contact angle reported by Sghaier et al. (2006), and $d_{b}=1 \mathrm{~mm}$, which is the largest bead size used in our experiments, this gives a maximum imbibition height $L_{i m b}=\left(P_{c} / \rho_{\ell} g\right)$ (Jurin's law) $\approx 4.5 \mathrm{~cm}$ (with $\varepsilon \approx 0.36, \rho_{\ell}$ is the density of the sodium chloride aqueous solution $\left.\approx 1200 \mathrm{~kg} \mathrm{~m}^{-3}\right)$. As mentioned before, the length $L_{s}\left(L_{s}=L-h\right)$ of the sample above the liquid level in the bath is equal to $22 \mathrm{~mm}$ in the second set of experiments and of the order of $10 \mathrm{~mm}$ in the first set of experiments at the beginning of the experiments. The total decreases of the liquid level in the bath due to the evaporation process are typically of the order of $0.2,0.9$ and $2.3 \mathrm{~mm}$ in our experiments for $\delta=2.5,7.5$ and $15 \mathrm{~mm}$, respectively; thus only slightly lower than at the beginning of experiments. As a result $L_{s}$ is always lower than $L_{i m b}$ and the porous medium remains fully saturated during an experiment. This is confirmed by the visual inspection of the sample surface when we stop the experiment, which shows that the solution still saturates the medium. The liquid level $h$ in the reservoir (see figure 2) is thus at any time such that the wick remains fully saturated owing to capillary action. It can be argued that this simple analysis does not take into account the additional pressure drop due to the viscous flow in the porous medium. It can be easily shown that this pressure drop is negligible compared with the variation in the hydrostatic pressure. 
The initial salt mass fraction throughout the sample when the experiment starts is uniform and is the same at the mass fraction imposed in the bottom reservoir, thus equal to $C_{0}=25 \%$. The sodium chloride aqueous solution is prepared by adding a certain mass of distilled water to a given mass of salt (e.g. $75 \mathrm{~g}$ of water for $25 \mathrm{~g}$ of salt). The duration of each experiment is in the range (1-3 weeks).

The evolution of the mass $m(t)$ of the wick-aqueous solution reservoir system is measured by placing the sample on a Mettler-Toledo PM 6100 precision scale with an accuracy of $0.01 \mathrm{~g}$. The solution mass loss is measured at 1 min intervals with data automatically stored on a computer.

The relative humidity and temperature are recorded during the experiment using a Rotronic Hygroclip SP05 probe set in the chamber. A Nikon D100 camera with a resolution of 3008 pixels $\times 2000$ pixels is set above the sample. Top images of the efflorescence developing on sample surface are recorded using the acquisition software Nikon Capture Control with a frequency between 2 and 12 images per hour depending on the value of $\delta$.

\section{Evaporation flux at the surface: influence of local disorder}

\subsection{Continuum computation of evaporation flux}

The evaporation flux distribution along the surface of the porous medium is first computed using the classical continuum approximation at the surface of the porous medium. The gas phase is a binary mixture made of air and water vapour. Neglecting heat transfer, the equations governing the transport of the vapour in the enclosure can then be expressed as

$$
\begin{gathered}
\frac{\partial \rho_{g}}{\partial t}+\nabla \cdot\left(\rho_{g} \boldsymbol{u}_{g}\right)=0 . \\
\rho_{g} \frac{\partial \boldsymbol{u}_{g}}{\partial t}+\rho_{g} \boldsymbol{u}_{g} \cdot \nabla \boldsymbol{u}_{g}=-\nabla P_{g}+\mu_{g} \nabla^{2} \boldsymbol{u}_{g}+\rho_{g} \boldsymbol{g} \\
\frac{\partial \rho_{g} X_{v}}{\partial t}+\nabla \cdot\left(\rho_{g} X_{v} \boldsymbol{u}\right)=\nabla \cdot\left(\rho_{g} D \nabla X_{v}\right),
\end{gathered}
$$

where $\rho_{g}$ is the density of the gas mixture, $\mu_{g}$ the gas viscosity, $P_{g}$ the gas pressure, $\boldsymbol{u}_{g}$ the gas velocity vector, $\boldsymbol{g}$ the gravitational acceleration, $D$ the binary molecular diffusion coefficient and $X_{v}$ the vapour mass fraction. Equations (3.1)-(3.3) are the gas-phase continuity equation, momentum conservation equation and vapour transport equation, respectively. It should be noted that $\rho_{g}$ and $\mu_{g}$ are functions of $X_{v}$, see, for instance, (Bird, Stewart \& Lightfoot 2002). Hence, there is in general a coupling between the vapour transport and the flow. To solve the above equations and determine the evaporation flux along the liquid saturated surface of the porous wick, boundary conditions should be supplemented. To this end, we first need to discuss the boundary condition to impose at an evaporative porous surface.

Consider a saturated porous surface, that is a porous surface where all surface pores are occupied by liquid. The basic question is whether the evaporation rate from a porous surface, that is a surface which is partially solid and partially liquid, is different (and a priori smaller) from the same surface fully covered by liquid. This problem was first addressed by Suzuki \& Maeda (1968), who showed that the evaporation rate from a porous surface was identical to that from the same surface assumed fully liquid provided the porous surface was sufficiently finely divided. In other terms, the average pore size and the mean distance between the pore openings at the surface 
must be sufficiently small compared with the characteristic size of the external mass transfer. Here this constraint can be expressed as $d_{b} \ll \delta_{e x t}$, where $\delta_{\text {ext }}$ is the external transfer characteristic length (the average thickness of the mass boundary layer in convective drying for example (e.g. Masmoudi \& Prat 1991) or the diameter of the surface in the case of diffusion driven evaporation from a disk (e.g. Picknett \& Bexon 1977)). This has been recently confirmed from 3D simulations (e.g. Veran-Tissoires et al. 2013). Hence, the remarkable result is that a porous surface behaves as a fully wetted surface provided that the bead size $d_{b}$ is sufficiently small compared with $\delta_{e x t}$. In our case where diffusion is the main transport mechanism of the vapour in the enclosure, an order of magnitude of $\delta_{\text {ext }}$ is therefore the diameter of the hollow cylinder $\delta_{e x t} \approx 38 \mathrm{~mm}$ and therefore $d_{b} \ll \delta_{e x t}$, noting however that the ratio $d_{b} / \delta_{\text {ext }}$ is small but not very small for the beads $1 \mathrm{~mm}$ in diameter.

The boundary condition at the surface is thus expressed as

$$
X_{v}=X_{v e}(C),
$$

where $X_{v e}$ is the equilibrium vapour mass fraction at a liquid/gas interface. For the almost-saturated sodium chloride aqueous solution considered in this paper, the water activity $a_{w}\left(a_{w}=p_{v}(C) / p_{v 0}\right.$, where $p_{v}(C)$ and $p_{v 0}$ are the vapour pressure for a sodium chloride aqueous solution and the vapour pressure for pure water, respectively) is less than for pure water and is close to 0.75 (Robinson 1945). Hence, $X_{v e}\left(C_{s a t}\right)=0.0198$ at $T=22^{\circ} \mathrm{C}$.

The evaporation flux at the porous medium surface is given by

$$
j=-\frac{\rho_{g} D}{1-X_{v e}} \nabla X_{v} \cdot \boldsymbol{n},
$$

where $\boldsymbol{n}$ is the unit normal vector at the surface.

For solving the flow equation (3.2), a no-slip boundary condition is imposed as regards the tangential component of the velocity vector whereas the normal component is expressed as

$$
\boldsymbol{u} \cdot \boldsymbol{n}=-\frac{D}{\left(1-X_{v e}\right)} \nabla X_{v} \cdot \boldsymbol{n} .
$$

Equation (3.6) reflects the fact that the evaporation process induces a flow within the gas phase. One can refer for instance to Carey (2008) for more details.

In the simplest situation, the external mass transfer is governed by diffusion. This is a correct assumption when the liquid is not too volatile and the evaporative surface is small. When the size of the evaporative surface is not sufficiently small, convective effects due to variation of density with gas mixture composition cannot be ignored. This can be understood from the expression of the Grashof number, which characterizes the competition between buoyancy effects and viscous effects $G r=g \beta\left(\rho_{v s a t}-\rho_{v \infty}\right) \Lambda^{3} / v_{g}^{2}$ where $\beta=-\left(1 / \rho_{g}\right)\left(\partial \rho_{g} / \partial \rho_{v}\right)_{T, P}, v_{g}$ is the gas kinematic viscosity, $\rho_{v s a t}$ the vapour concentration at the surface and $\rho_{v \infty}$ the vapour concentration in ambient (surrounding) medium. The Grashof number varies as $\Lambda^{3}$, where $\Lambda$ is the characteristic length of the surface $\left(\Lambda=2 r_{w}\right.$ in our case). The high value of the exponent (3) explains why free convection effects cannot generally be neglected as soon as the size of the surface is typically greater than a few millimetres. This is illustrated from the simple experiment sketched in figure 2 . The evaporation rate $J$ is deduced from the evolution of the mass $m(t)$ of the wick-aqueous solution reservoir system. Hence, $J=-\mathrm{d} m / \mathrm{d} t$. Then the evaporation 
rate is computed from the numerical solution of (3.1)-(3.3) supplemented by the boundary condition $X_{v} \approx X_{v e}\left(C_{0}\right) \approx X_{v e}\left(C_{s a t}\right)$ at the porous medium surface and the condition $X_{v}=0.066 X_{v e}(0)$ at the surface of the LiBr solution. A zero-flux condition is imposed at other surfaces bounding the gas phase within the enclosure. The same computation was also made assuming a purely diffusive transport, that is, by solving (3.3) with $\boldsymbol{u}_{g}=0$ and $\rho_{g}$ constant. In both cases the evaporation rate is computed from

$$
J=\int_{A} j_{e} \mathrm{~d} S=-\int_{A} \frac{\rho_{g} D}{1-X_{v e}} \nabla X_{v} \cdot \boldsymbol{n} \mathrm{d} A
$$

where $A$ is the evaporative surface of the porous medium (i.e. the top disk-like surface of the porous medium). The commercial simulation software COMSOL Multiphysics was used to obtain all of the numerical solutions discussed in this section. Simulations were run using cylindrical coordinates on the gas domain of the experimental set-up, with triangle meshes of approximately 45000 elements. Meshes are finer at the porous medium surface.

This was done for the three different values of $\delta$ so as to vary the evaporation rate. Reducing $\delta$ increases the overall evaporation rate (the external mass transfer resistance due to transfers between the porous medium surface and the hollow cylinder entrance is reduced) and modifies the evaporation flux distribution over the surface. Increasing $\delta$ reduces the evaporation rate (the resistance due to transfers between the porous medium surface and the hollow cylinder entrance is increased) and makes the local evaporation flux distribution increasingly uniform. The results are summarized in table 1. As can be seen, the purely diffusive computation leads to discrepancies with the experimental results. Although the agreement is not perfect, the computation including the free convection effect leads to better results. More details can be found in Veran-Tissoires (2011). Figure 3 shows the corresponding distributions of evaporation flux at the surface. As can be seen, the evaporation flux is greater at the periphery when the distance $\delta$ is sufficiently small and tends to be increasingly uniform as $\delta$ increases. The consideration of free convection effects leads to slightly more uniform distributions compared with those obtained with the purely diffusive approximation. It can be also noted from table 1 that the evaporation flux is sensitive to parameter $L_{s}$, i.e. to the curvature and position of menisci at the interface, at least for the $1 \mathrm{~mm}$ beads. A greater $L_{s}$ means a greater curvature of menisci and a mean position of menisci a bit more inside the spaces between the beads at the surface.

\subsection{Influence of local disorder}

The analysis of the distribution of the precipitation onset points at the porous medium surface requires studying the possible impact of the pore-scale heterogeneities at the porous medium surface on the evaporation flux distribution. To gain insights into the distribution, we consider the simple model surface sketched in figure 4. The circular porous surface is of radius $r_{w}$. Pore openings are located at the nodes of a square mesh. The distance between two nodes (mesh spacing) is $a$ ( $a \approx d_{b}$ in a packing of monodisperse beads). The diameter $d_{o}$ of openings is distributed randomly in the range $\left[d_{\text {omin }}, d_{\text {omax }}\right]$ according to a Gaussian distribution with the following parameters: mean value of $0.3 a$, standard deviation of $0.04 a, d_{\text {omin }}=0.18 a$ and $d_{\text {omax }}=0.42 a$. This model surface is consistent with the cubic pore network model presented in $\S 4.2$. The computation is performed as follows. Free convection effects are neglected within the reduced computational domain considered. The later is the domain occupied by the gas phase above the porous surface in the hollow cylinder. This domain is therefore 
$J_{\text {exp }}\left(\mathrm{kg} \mathrm{s}^{-1}\right)\left(d_{b}=1 \mathrm{~mm}\right)$

$J_{\text {exp }}\left(\mathrm{kg} \mathrm{s}^{-1}\right)\left(d_{b}=1 \mathrm{~mm}\right)$

$J_{\exp }\left(\mathrm{kg} \mathrm{s}^{-1}\right)\left(d_{b}=100-125 \mu \mathrm{m}\right)$

$J_{\text {exp }}\left(\mathrm{kg} \mathrm{s}^{-1}\right)\left(d_{b}=50-62 \mu \mathrm{m}\right)$

$J_{\exp }\left(\mathrm{kg} \mathrm{s}^{-1}\right)\left(d_{b}=5-50 \mu \mathrm{m}\right)$

$J_{\text {num }}\left(\mathrm{kg} \mathrm{s}^{-1}\right)$ pure diffusion

Relative error (\%)

$J_{\text {num }}\left(\mathrm{kg} \mathrm{s}^{-1}\right)$ free convection + diffusion

Relative error $(\%)$

$$
\begin{array}{cccc}
L_{s}(\mathrm{~mm}) & \delta=2.5 \mathrm{~mm} & \delta=7.5 \mathrm{~mm} & \delta=15 \mathrm{~mm} \\
\approx 10 & 2.7 \times 10^{-8} & 2.37 \times 10^{-8} & 2.02 \times 10^{-8} \\
22 & - & - & 1.80 \times 10^{-8} \\
22 & - & - & 1.74 \times 10^{-8} \\
22 & - & - & 1.75 \times 10^{-8} \\
22 & - & - & 1.9 \times 10^{-8} \\
& 1.92 \times 10^{-8} & 1.53 \times 10^{-8} & 1.21 \times 10^{-8} \\
& 29 & 35 & 30-40 \\
& 2.72 \times 10^{-8} & 2.08 \times 10^{-8} & 1.55 \times 10^{-8} \\
& 0.7 & 12 & 10-23
\end{array}
$$

TABLE 1. Comparison between computed and measured evaporation rates. The relative error is between experimental results (beads $1 \mathrm{~mm}$ in diameter) and the computed ones. For the beads $(5-50 \mu \mathrm{m})$ and $(50-62 \mu \mathrm{m})$, the experimental evaporation rate in the table is that at the beginning of evaporation before the significant development of efflorescence (see $\S 6)$.

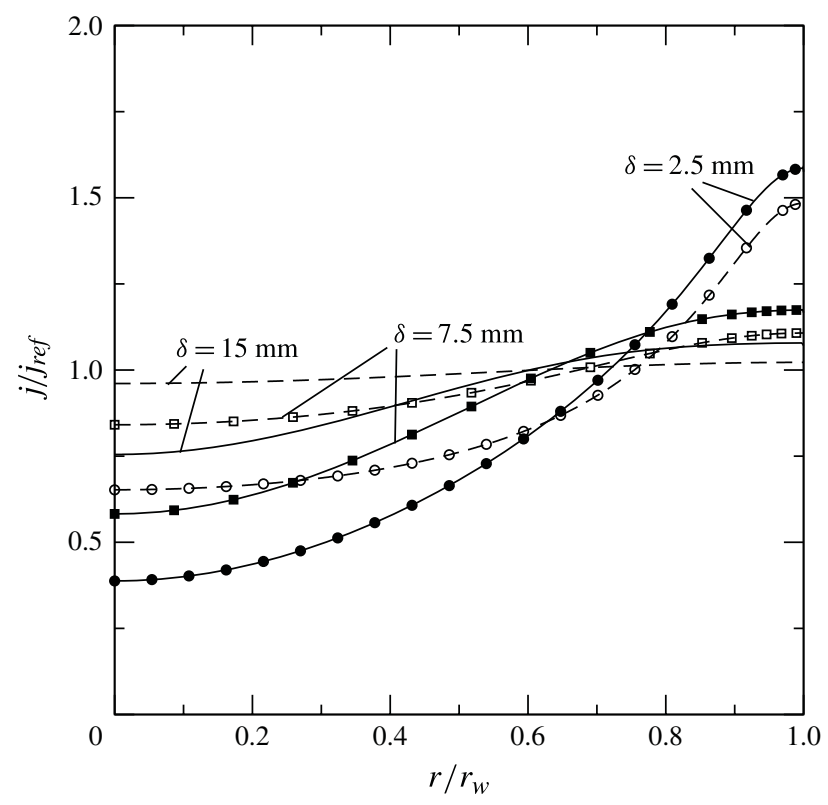

FIGURE 3. Variation of evaporation flux at the surface of porous medium as a function of radial position for the three values of $\delta$. The thick lines are for the computations taking into free convection effects whereas the thin lines are for the purely diffusive model. The reference evaporation flux $j_{r e f}$ depends on $\delta$ and is given by $J(\delta) /\left(\pi r_{w}^{2}\right)$ where $J(\delta)$ is the computed evaporation rate over the wick top surface.

the cylindrical domain located between the plane $z=L$ and $z=L+\delta$. In the dilute limit considered here, the equation governing the mass fraction of vapour in the computation domain (see figure 2) reads,

$$
\Delta X_{v}=0 .
$$




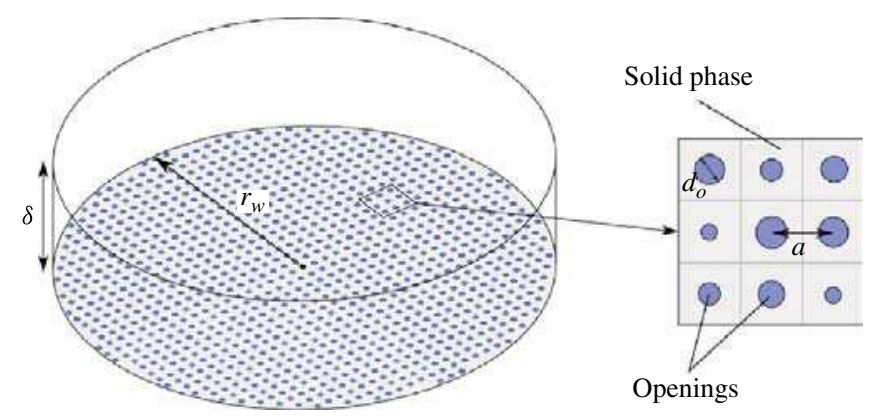

FIGURE 4. (Colour online) Sketch of model porous surface considered to evaluate the influence of surface opening disorder on the evaporation rate from surface openings. The aperture of openings is randomly distributed.

At the porous surface, the boundary conditions read,

$$
\begin{gathered}
\nabla X_{v} \cdot \boldsymbol{n}=0 \quad \text { at } \Omega_{s} \\
X_{v}=X_{v e}\left(C_{\text {sat }}\right) \quad \text { at } \Omega_{o},
\end{gathered}
$$

where $\Omega_{s}$ and $\Omega_{o}$ represent the solid part of the porous medium surface and the surface of openings, respectively. Note that the liquid surface at the entrance of openings is assumed to be flat for simplicity. Equation (3.9) expresses that there is no evaporation flux over the solid part of the porous surface. On the lateral side (corresponding to the hollow cylinder inner wall), a zero flux condition is imposed $\left(\nabla X_{v} \cdot \boldsymbol{n}=0\right)$, whereas the boundary condition over the plane located at $z=L+\delta$ is expressed as

$$
\nabla X_{v} \cdot \boldsymbol{n}=\nabla X_{v_{c}} \cdot \boldsymbol{n} \quad \text { at } z=L+\delta
$$

where $\nabla X_{v_{c}}$ is the gradient computed using the continuum approach when free convection effects in the enclosure are neglected, see $\$ 3.2$. According to the results presented in $\$ 3.2$, it would be of course desirable to take into account the free convection effects but this would quite considerably increase the computational effort. The above problem was solved using the free open-source CFD software OpenFOAM ${ }^{\circledR}$. The gas domain is decomposed into an union of rectangular cuboids. Each cuboid contains an opening and is finely meshed by 840000 hexahedron elements. Then the evaporation rate $J_{o}$ from each surface opening is computed.

The results obtained for one realization of the surface and the three values of distance $\delta$ are depicted in figure 5. The remarkable result is that there is not a one-to-one correspondence between the evaporation rate at the entrance of an opening and its diameter. There are two main effects. The first is obvious and is the peripheral effect (figure 3), which is marked for $\delta=2.5 \mathrm{~mm}$ and $\delta=7.5 \mathrm{~mm}$. The evaporation rate from an opening of small radius at the periphery can be greater than the evaporation rate from a larger opening located closer to the centre of the surface. The second is subtler and results from the variation in the opening size of neighbour openings. For example, the evaporation rate from an opening whose neighbours are on average of small diameter is greater than the evaporation rate from an opening of same diameter and located at the same radial position whose neighbour openings are on average of greater diameter. This is illustrated in figure 6 . This effect is referred to as a cooperation effect between neighbour openings. The 

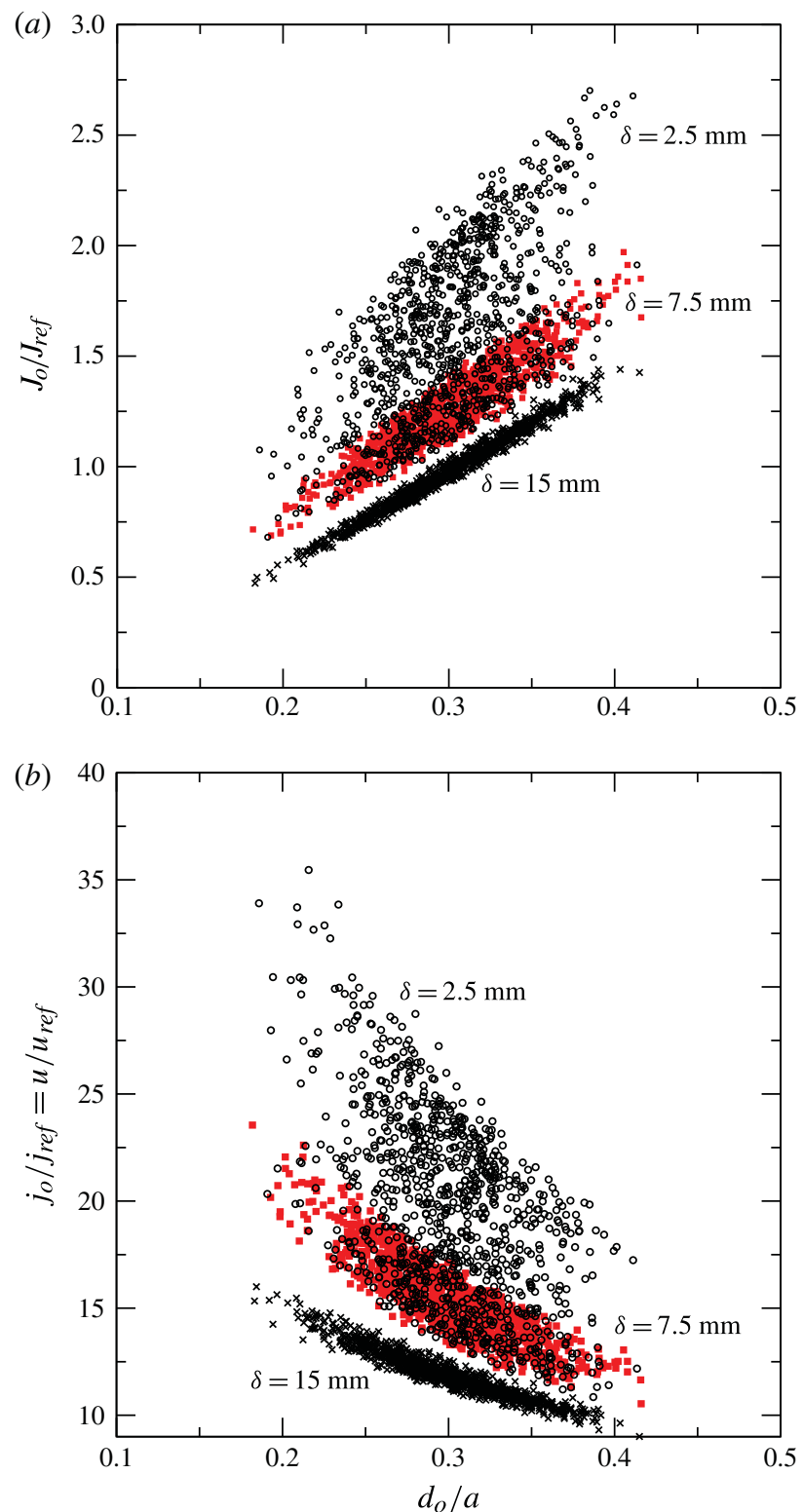

FIGURE 5. (Colour online) (a) Dimensionless evaporation rate at the entrance of each opening as a function of opening diameter, $(b)$ mean evaporation flux at the entrance of each opening as a function of opening diameter; the reference evaporation flux $j_{\text {ref }}$ is given by $j_{\text {ref }}=J_{15 \text { num }} /\left(\pi r_{w}^{2}\right)$ where $J_{15 \text { num }}$ is the evaporation rate computed with the continuum model assuming a purely diffusive vapour transfer for $\delta=15 \mathrm{~mm}$ (as reported in table 1, $J_{15 \text { num }}=1.21 \times 10^{-8} \mathrm{~kg} \mathrm{~s}^{-1}$ ). The average velocity $u$ in an interfacial throat is given by $u=j_{o} / \rho_{\ell}$. Thus, $j_{o} / j_{\text {ref }}=u / u_{\text {ref }}$. The reference evaporation rate $J_{\text {ref }}$ is computed as $J_{r e f}=$ $j_{\text {ref }} a^{2}$. Here $\delta=2.5 \mathrm{~mm}(\circ), \delta=7.5 \mathrm{~mm}(\boldsymbol{\square}), \delta=15 \mathrm{~mm}(\times)$. The dispersion in the data is due to the peripheral effect and the spatial variability in the opening diameter. 


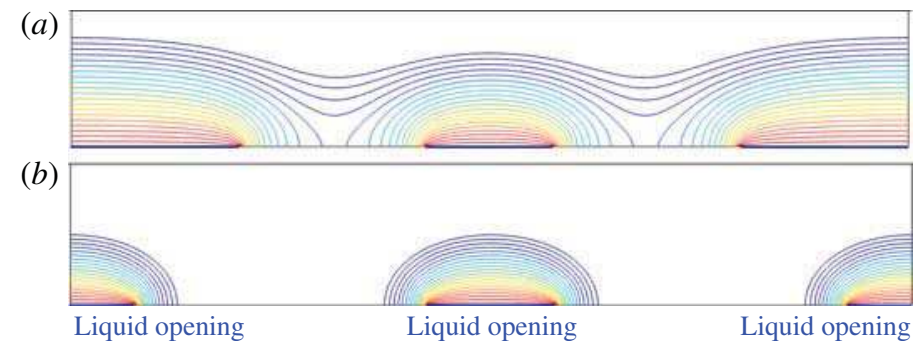

FIGURE 6. (Colour online) Water vapour isoconcentration lines from three discrete openings at the surface. The normalized vapour concentration is set at one at the liquid surface and zero far from the heterogeneous surface. The normalized concentration associated with each isoline varies from 0.995 to 0.9 with steps of 0.005 . In the image on top, the two neighbour side openings have a larger surface area compared with the central opening (ratio 8/3). In the image on the bottom, the surface area of the two neighbour side openings is the same as the surface area of the central opening. One can clearly see from the isoconcentration lines that the evaporation fluxes from the central opening are higher in the second case. The evaporation rate from the central opening surrounded by the larger openings is here 1.33 times smaller than when the central opening and the neighbour openings are of the same surface area.

essential point is that there are spatial fluctuations in the evaporation rate from one opening to the other at the surface. This is illustrated in figure 5, which also shows that not only the variations due to the peripheral effect but also the fluctuations due to the cooperation/neighbouring effect diminish as the distance $\delta$ is increased. The computation were also performed for the case $\delta=15 \mathrm{~mm}$ with a spatially uniform boundary condition at $L+\delta$ (corresponding to the mean evaporation flux deduced from the continuum computation). The results are very close to those shown in figure 5. This is an indication that the relatively good correlation with the opening size for the case $\delta=15 \mathrm{~mm}\left(J_{o} \propto d_{o}\right)$ has nothing to do with the peripheral effect.

Of particular interest is the distribution of the mean evaporation flux $j_{o}=J_{o} /\left(\pi d_{o}^{2} / 4\right)$ at the surface of the openings because there is a direct link between the mean velocity $u$ induced in the liquid solution right below the opening and the evaporation flux at the entrance of the interfacial throats, $u=j_{o} / \rho_{\ell}$.

The fact that the evaporation flux distribution should be properly estimated can be illustrated as follows. Suppose that we determine the evaporation rate at the surface of the interfacial bonds from the simple mapping of the average flux

$$
J_{o m}(r, \delta)=j(r, \delta) a^{2}
$$

where $j(r, \delta)$ is the evaporation flux distribution deduced from the continuum approach (figure 3). Consider as an example, the case $\delta=15 \mathrm{~mm}$. The results are shown in figure 7. As can be seen, the numerical computation of the evaporation rate from the discrete computation leads to quite different results from the simple mapping given by (3.12). Of particular interest is the evolution of the interfacial velocities $u=J_{o} /\left(\pi d_{o}^{2} / 4\right) / \rho_{\ell}$. As can be seen from figure $7(b)$, the variability of the interfacial velocity is much less when the cooperation effect between neighbour openings is taken into account.

A last effect must also be considered. It is well known that the porosity of a random packing of spherical particles is greater in the vicinity of the solid wall (e.g. Vafai 1984). Following Vafai (1984), the porosity variation near the solid wall (porosity wall 

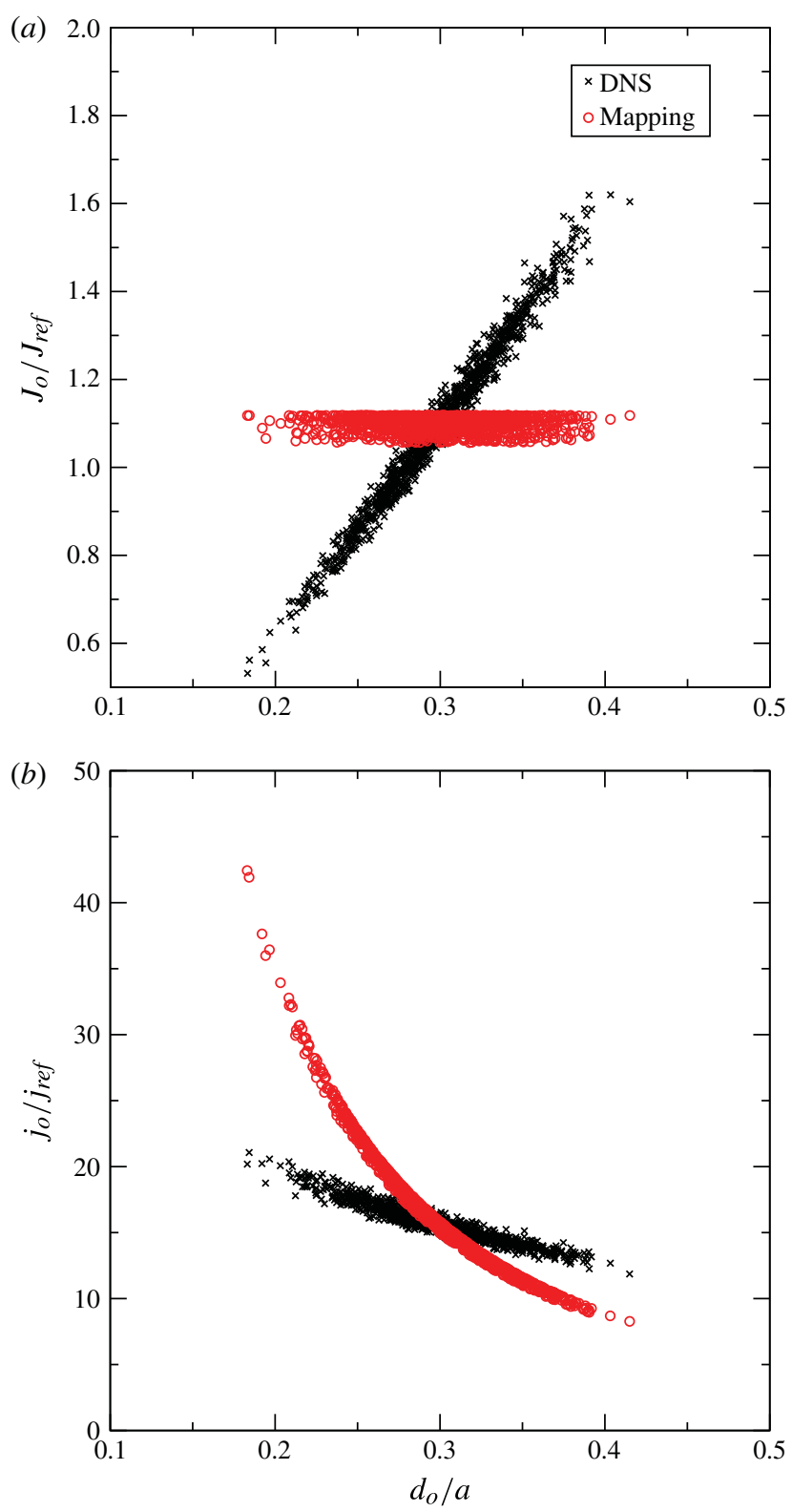

FiguRE 7. (Colour online) Comparison of evaporation rate and mean evaporation flux at the entrance of each opening of the model surface (figure 4) between the full three-dimensional computation (direct numerical simulation) and the simple mapping corresponding to (3.12): (a) dimensionless evaporation rate at the entrance of each opening as a function of opening diameter; $(b)$ mean evaporation flux at the entrance of each opening as a function of opening diameter. The reference evaporation flux $j_{r e f}$ is given by $j_{r e f}=J_{t o t} /\left(\pi r_{w}^{2}\right)$ where $J_{t o t}$ is the global evaporation rate leaving the evaporation surface. The average velocity $u$ in an interfacial throat is given by $u=j_{o} / \rho_{\ell}$. Thus $j_{o} / j_{\text {ref }}=u / u_{\text {ref }}$. The reference evaporation rate $J_{r e f}$ is computed as $J_{r e f}=j_{r e f} a^{2}$. 
effect) can be expressed as

$$
\varepsilon\left(r_{w}-r\right)=\varepsilon_{0}\left(1+\alpha \exp \left(\beta \frac{\left(r_{w}-r\right)}{d_{b}}\right)\right)
$$

where $\varepsilon_{0}$ is the porosity far from the wall and $\alpha$ and $\beta$ two numerical factors $(\alpha=0.98, \beta=-2)$.

As a result, it is surmised that the size of the openings should be greater on average at the periphery. This effect has been taken into account by increasing the size of the openings at the periphery in accordance with (3.13). This leads to the results depicted in figure 8. The comparison with the results reported in figure 5, shows the greater evaporation rates at the periphery resulting from both the increase in the opening sizes (porosity wall effect) and the flux peripheral effect (figure 3). As shown in figure 8(b), the porosity wall effect leads to lower evaporation flux, and thus lower interfacial velocities in the near-wall region (peripheral region of the surface) compared with the situation when this effect is not considered.

\section{Ion distributions at the surface of the porous medium}

\subsection{Experiments}

As illustrated in figure 9, the experiment leads to the crystallization of salt at the surface of porous medium. The images shown in figure 9 were obtained for a packing of monodisperse beads $1 \mathrm{~mm}$ in diameter and the three different distances $\delta=2.5$, 7.5 and $15 \mathrm{~mm}$. The effect of bead size is discussed in $\S 5$. As can be seen, the efflorescence does not form a crust covering all of the surface of the porous medium but forms a set of well-individualized structures referred to as patches. This type of efflorescence, frequently observed in nature or at the surface of porous stones, is therefore called 'patchy'. One can distinguish two main phases. During the first phase the surface of the porous medium is free of efflorescence. This phase is relatively short, $\sim 5 \mathrm{mn}, 45 \mathrm{mn}$ and $8 \mathrm{~h}$ for the experiments with $\delta=2.5,7.5$ and $15 \mathrm{~mm}$, respectively, since the initial salt concentration in the system is close to the saturation concentration. As discussed below, the salt mass fraction increases constantly at the interface during this phase. When the crystallization mass fraction $C_{c r i s}$ is reached somewhere at the surface, crystals form at the surface. In general, it is expected that $C_{c r i s}>C_{\text {sat }}$. Defining here the supersaturation as $\sigma=C_{c r i s} / C_{\text {sat }}$, it is thus expected that the onset of crystallization occurs for a certain degree of supersaturation. It is often considered that supersaturation effects are weak for $\mathrm{NaCl}$ and therefore that the crystallization mass fraction is close to the saturation mass fraction $\left(C_{s a t}=26.4 \%\right)$, Chatterji (2000), Pel, Huinink \& Kopinga (2002) and Espinosa-Marzal \& Scherer (2010). However, recent experiments of crystallization in capillary tubes (e.g. Shahidzadeh \& Desarnaud 2012) or at the surface of porous media (e.g. Hidri et al. 2013), indicate that the supersaturation can be noticeable. As we will see invoking supersaturation effect is also necessary to obtain a good agreement between the simulations and the experiments discussed in the present paper as regards the time of first precipitation. For the moment, we therefore simply state that crystallization is expected to start when the ion mass fraction $C \approx C_{\text {cris }}$ somewhere at the surface with $C_{c r i s}>C_{\text {sat }}$.

In the case of the experiment illustrated in figure $9(c)(\delta=15 \mathrm{~mm})$, precipitation starts at only one point of the surface. Then after a noticeable time $(\sim 13 \mathrm{~h}$ in our experiment), other precipitation spots can be observed. However, several precipitation 

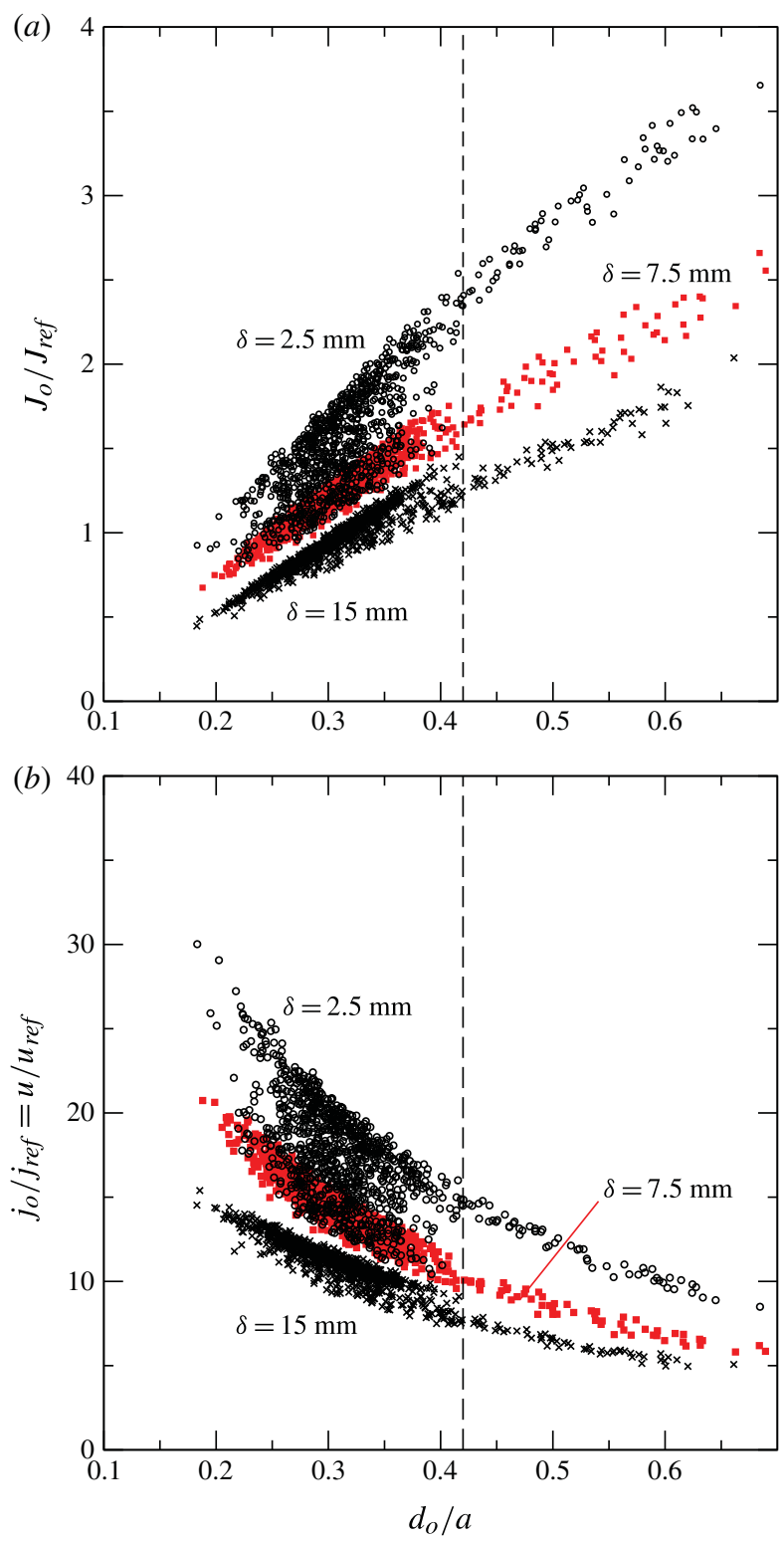

FIGURE 8. (Colour online) Influence of peripheral porosity increase on local evaporation. The results for the opening sizes greater than 0.42 (vertical dashed line) correspond to the peripheral region of greater porosity (see the text): $(a)$ dimensionless evaporation rate at the entrance of each opening as a function of opening diameter; $(b)$ mean evaporation flux at the entrance of each opening as a function of opening diameter; the reference evaporation flux $j_{\text {ref }}$ is given by $j_{\text {ref }}=J_{15 \text { num }} /\left(\pi r_{w}^{2}\right)$ where $J_{15 \text { num }}$ is the evaporation rate computed with the continuum model assuming a purely diffusive vapour transfer for $\delta=$ $15 \mathrm{~mm}$ (as reported in table $1, J_{15 \text { num }}=1.21 \times 10^{-8} \mathrm{~kg} \mathrm{~s}^{-1}$ ). The average velocity $u$ in an interfacial throat is given by $u=j / \rho_{\ell}$. Thus, $j / j_{\text {ref }}=u / u_{\text {ref }}$. The reference evaporation rate $q_{\text {ref }}$ is computed as $q_{\text {ref }}=j_{r e f} a^{2}$. Here $\delta=2.5 \mathrm{~mm}(0), \delta=7.5 \mathrm{~mm}(\mathbb{\square}), \delta=15 \mathrm{~mm}$ $(\times)$. The dispersion in the data is due to the spatial variability in the opening diameter, the peripheral effect and the porosity wall effect. 
(a)
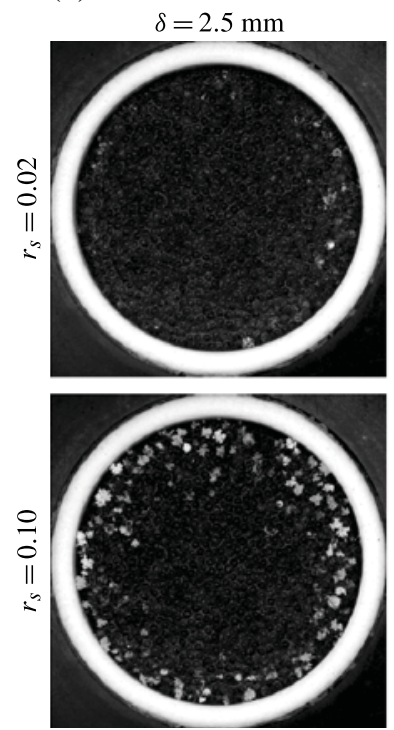

(d)

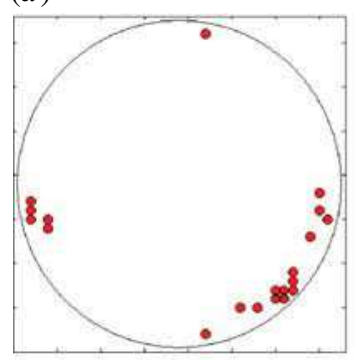

( $g$ )

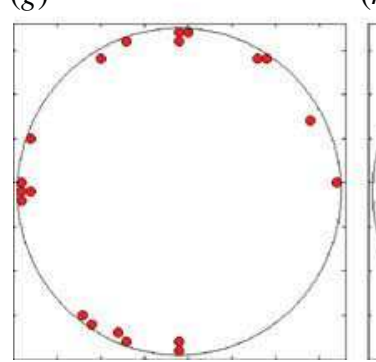

(b)
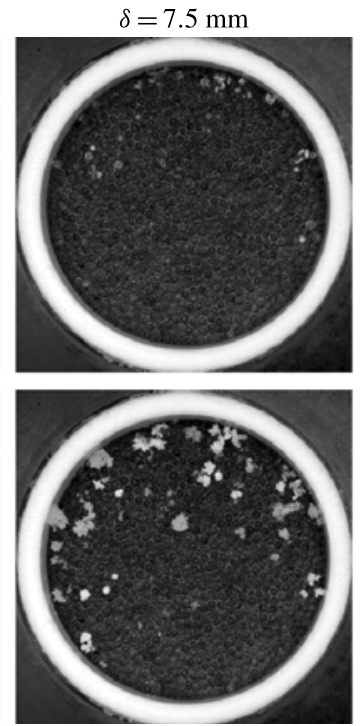

$(e)$

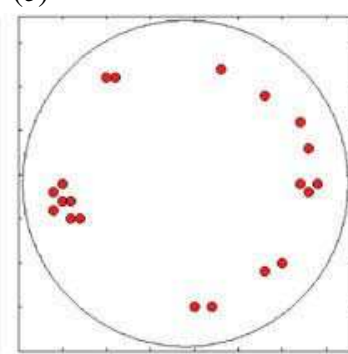

(h)

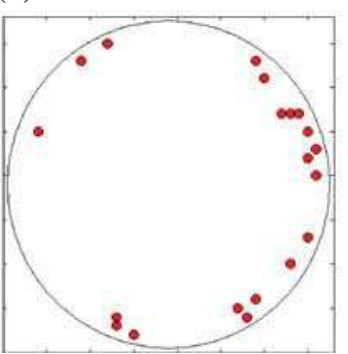

(c)
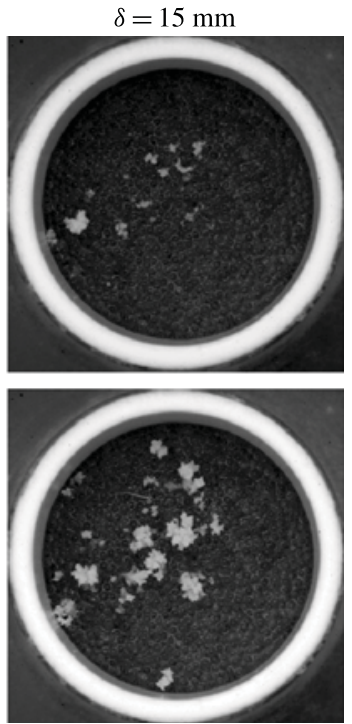

$(f)$

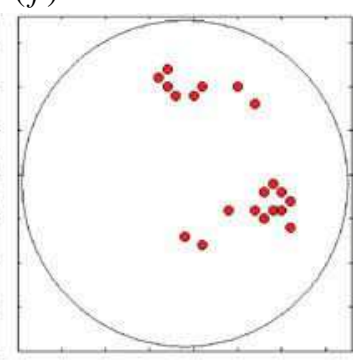

(i)

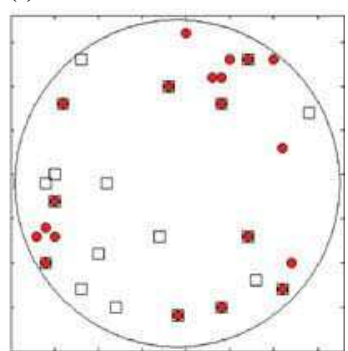

FIGURE 9. (Colour online) $(a-c)$ Images of the top surface of porous medium a little after the beginning of precipitation $\left(r_{s}=0.02\right)$ and later when the projected area of efflorescence is equal to $10 \%$ of the area of the porous medium surface $\left(r_{s}=0.1\right)$ for the three distances $\delta$. Efflorescence structures in white are clearly visible; $r_{s}=A_{s} / A$ is the ratio between the surface area $A_{s}$ covered by efflorescence on a $2 \mathrm{D}$ image and the area $A$ of the porous medium surface. $(d-f)$ Position of 20 greater salt concentration maxima at the surface from full pore network simulations. $(g-i)$ Position of 20 greater salt concentration maxima at the surface from pore network simulations without the near-wall porosity variation (the empty squares in figure $9(i)$ correspond to the 20 throats of higher evaporation flux). 
spots can appear almost simultaneously for lower $\delta$. The remarkable result illustrated in figure 9 is that the number of crystallized spots is small and still more remarkable is that the number of individualized crystallized salt growing structures remains small, much less than the number of pore openings at the surface, during the entire experiment duration (the number of pore openings is roughly $N_{o} \approx\left(\pi r_{w}^{2} / d_{b}^{2}\right) \approx 1100$ ). This is obvious from figure $9(c)$ and will be characterized in more detail in $\S 6$. In this section, we focus on what happens during the first phase up to the occurrence of first crystals, that is on the ion distribution at the surface of the porous medium before the onset of crystallization. The second remarkable result emerging from figure 9 is that the distribution of precipitation discrete spots greatly depends on the distance $\delta$. A clear peripheral distribution is observed when the distance $\delta$ is sufficiently small.

\subsection{Continuum approach}

\subsubsection{Ion transport volume-averaged equation}

Within the framework of the classical continuum approach to porous media (e.g. Bear 1972), the equation governing the ion transport within the porous medium reads

$$
\frac{\partial \rho_{\ell} \varepsilon C}{\partial t}+\nabla \cdot\left(\rho_{\ell} \varepsilon \boldsymbol{U} C\right)=\nabla \cdot\left(\rho_{\ell} \varepsilon D_{s}^{*} \nabla C\right)
$$

where $C$ is the mass fraction of dissolved salt. Here $D_{s}^{*}$ is the effective diffusive coefficient of the dissolved salt in the liquid, $\rho_{\ell}$ the solution density, $\varepsilon$ the porosity of the porous medium and $\boldsymbol{U}$ the average interstitial velocity of the solution. For a random packing of spherical particles, $D_{s}^{*} \approx \varepsilon^{0.4} D_{s}$ (Kim, Ochoa \& Whitaker 1987), where $D_{s}=1.3 \times 10^{-9} \mathrm{~m}^{2} \mathrm{~s}^{-1}$ is the ion diffusion coefficient. The initial condition is $C=C_{0}$ throughout the sample. Using cylindrical coordinates, the boundary conditions can be expressed as

$$
\begin{aligned}
& C=C_{0} \text { at } z=0 . \\
& \left(\rho_{\ell} \varepsilon \boldsymbol{U} C-\rho_{\ell} \varepsilon D_{s}^{*} \nabla C\right) \cdot \boldsymbol{n}=0 \text { at } z=L \text { for all } r \text { and at } r=r_{w} \text { for all } z .
\end{aligned}
$$

The zero flux boundary condition (4.3) expresses that the dissolved salt cannot leave the porous medium before the onset of crystallization.

For simplicity, the solution density $\rho_{\ell}$ and its viscosity are considered as constant. This implies that the flow problem is decoupled from the dissolved salt transport problem.

The above problem is cast into dimensionless form using $C_{\text {cris }}, L, t_{\text {ref }}=L / \bar{U}$, as the characteristic ion mass fraction, length and time, respectively, with $\bar{U}=J / A / \varepsilon / \rho_{\ell}$, where $A=\pi r_{w}^{2}$ is the porous medium surface area. Using the superscript, to define the dimensionless variables, this gives

$$
\begin{gathered}
P e\left(\frac{\partial C^{\prime}}{\partial t^{\prime}}+\boldsymbol{U}^{\prime} \cdot \nabla C^{\prime}\right)=\nabla \cdot \nabla C^{\prime} \\
\left(P e \boldsymbol{U}^{\prime} C^{\prime}-\nabla C^{\prime}\right) \cdot \boldsymbol{n}=0 \text { at } z^{\prime}=1 \text { or } r^{\prime}=r_{w} / L C^{\prime}=C_{0} / C_{c r i s} \text { at } z^{\prime}=0
\end{gathered}
$$

where $P e=\left(\bar{U} L / D_{s}^{*}\right)$ is the Péclet number. To solve the above problem, the velocity field in the porous medium must be known. Using Darcy's law, the boundary value problem describing the flow in the porous medium is given by (after decomposition of the pressure according to $P=P_{v i s}+\rho_{\ell} g z$ ),

$$
\begin{gathered}
\boldsymbol{\nabla} \cdot \boldsymbol{V}=0 \\
\boldsymbol{V}=-\frac{K}{\mu} \nabla P_{v i s}
\end{gathered}
$$


where $\boldsymbol{V}$ is the filtration velocity, $\boldsymbol{V}=\varepsilon \boldsymbol{U}, K$ the porous medium permeability and $\mu$ the liquid solution viscosity. The boundary conditions read $P_{v i s}=P_{0}$ (arbitrary constant) at $z=0, \boldsymbol{V} \cdot \boldsymbol{n}=0$ on the porous column lateral side ( $\boldsymbol{n}$ is here the unit vector normal to the inner surface of cylinder containing the porous medium). At the porous medium surface, the evaporation flux is balanced by the liquid flow coming from the porous medium,

$$
\rho_{\ell} V_{z}=\rho_{\ell} \varepsilon U_{z}=j(r, \delta) \quad \text { at } z=L .
$$

Using $P_{\text {ref }}=(\mu \bar{U} / \varepsilon L)$ as the reference pressure, the problem giving the filtration velocity field is expressed in dimensionless form as

$$
\begin{gathered}
\nabla \cdot \boldsymbol{v}^{\prime}=0 \\
\boldsymbol{v}^{\prime}=-\frac{K}{L^{2}} \nabla P_{v i s}^{\prime} \\
v_{z}^{\prime}=\frac{j(r, \delta)}{\rho_{\ell} \bar{U}} \quad \text { at } z^{\prime}=1, \quad P_{v i s}^{\prime}=P_{0}^{\prime} \text { (arbitrary constant) at } z^{\prime}=0, \quad \boldsymbol{v}^{\prime} \cdot \boldsymbol{n}_{r}=0 .
\end{gathered}
$$

As discussed in previous papers (e.g. Puyate \& Lawrence 1998; Puyate et al. 1998; Puyate \& Lawrence 1999; Huinink et al. 2002; Sghaier et al. 2007; Guglielmini et al. 2008; Veran-Tissoires et al. 2012a), the distribution of the ions depends on the competition between advection, which tends to transport the ions towards the porous medium surface, and diffusion, which tends to make the ion distribution uniform. The Péclet number $P e$ characterizes this competition. Under usual evaporation conditions, the Péclet number is greater than one. In our experiments the Péclet number, which can thus be expressed as $P e=J L / \rho_{\ell} A \varepsilon D_{s}^{*}$, is equal to 3, 2.4 and 1.7 for $\delta=2.5,7.5$ and $15 \mathrm{~mm}$, respectively. When the Péclet number is of the order or greater than one, the advective effect is sufficient to lead to the accumulation of ions at the evaporative surface. The crystallization concentration is thus first reached at the surface, which leads to the formation of efflorescence.

First insights can be gained from the solution to the above problem in one dimension.

\subsubsection{One-dimensional solution}

When the medium is homogeneous (negligible spatial variations in $K$ or $\varepsilon$ ) and the evaporation spatially uniform at the surface ( $\delta \approx 15 \mathrm{~mm}$ or greater in our experiments), the ion transport problem greatly simplifies. First the filtration velocity is spatially homogeneous and simply given from (4.9) and (4.11) by

$$
V^{\prime}=(0,0, \varepsilon) .
$$

The typical distribution of ion within the sample is sketched in figure 10. Owing to the advection effect, the ion mass fraction is in fact uniform, equal to the mass fraction $C_{0}$ in the region of the sample adjacent to the bottom reservoir. As illustrated in figure 10, the salt mass fraction gradients are significant only in a region of high salt mass fractions of size $\xi(t)$ adjacent to the porous medium surface. The size of this region increases with time.

To solve the 1D versions of (4.4) and (4.5), we use a method similar to that developed in Guglielmini et al. (2008) for the drying problem. The method is based 


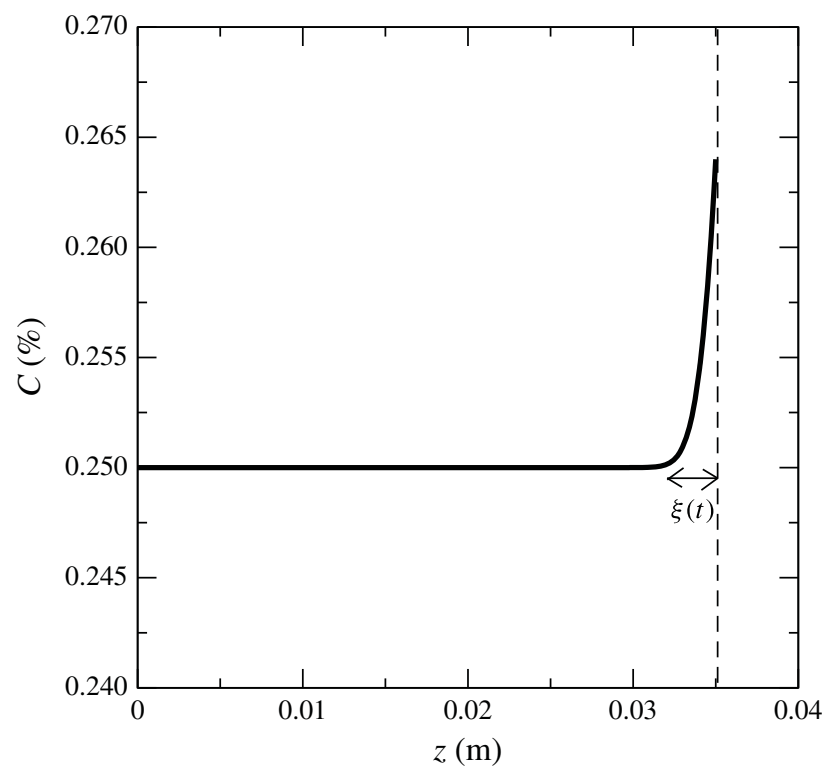

FIGURE 10. Typical ion distribution within the sample. The vertical dashed line indicates the position of the sample top surface.

on the observation that the distribution of the ions in the gradient region of size $\xi(t)$ can be considered as quasi-steady. In our case, this quasi-steady distribution reads $C=C_{0} \exp \left(P e Z^{\prime}\right)$, where $Z^{\prime}=0$ in $z^{\prime}=1-\xi^{\prime}(t)$. We then express the extra mass of salt contained in the gradient region as a result of the advection effect as

$$
\frac{M_{e x}}{\rho_{\ell} A L \varepsilon C_{0}}=\left[\int_{0}^{\xi^{\prime}(t)} \exp \left(P e Z^{\prime}\right) \mathrm{d} Z^{\prime}-\xi^{\prime}(t)\right] .
$$

Then we express that the variation of this mass is due to the salt entering into the porous medium at is bottom (where the salt concentration is $C_{0}$ as long as $\xi^{\prime}(t)<1$ ),

$$
\frac{\mathrm{d} M_{e x}}{\mathrm{~d} t}=\rho_{l} A C_{0} \varepsilon \bar{U} .
$$

Combining (4.13) and (4.14) and using as dimensionless time $\tau=t D_{s}^{*} / L^{2}$ give the equation governing the evolution of $\xi^{\prime}(t)$

$$
\exp \left(P e \xi^{\prime}\right)-P e \xi^{\prime}=P e^{2} \tau+1, \quad \xi^{\prime}=0 \text { at } \tau=0 .
$$

The associated salt mass fraction profile is given by $C^{\prime}=C_{0} / C_{c r i s}$ for $0 \leqslant z^{\prime} \leqslant 1-$ $\xi^{\prime}(\tau)$ and $C^{\prime}=\left(C_{0} / C_{c r i s}\right) \exp \left(\operatorname{Pe}\left(z^{\prime}-1+\xi^{\prime}\right)\right)$ for $1-\xi^{\prime}(\tau) \leqslant z^{\prime} \leqslant 1$.

The general solution to (4.15) is given by

$$
\xi^{\prime}=-\frac{W\left(-1,-\exp \left(-P e^{2} \tau-1\right)\right)+1+P e^{2} \tau}{P e},
$$

where $W$ is the Lambert $W$ function. 


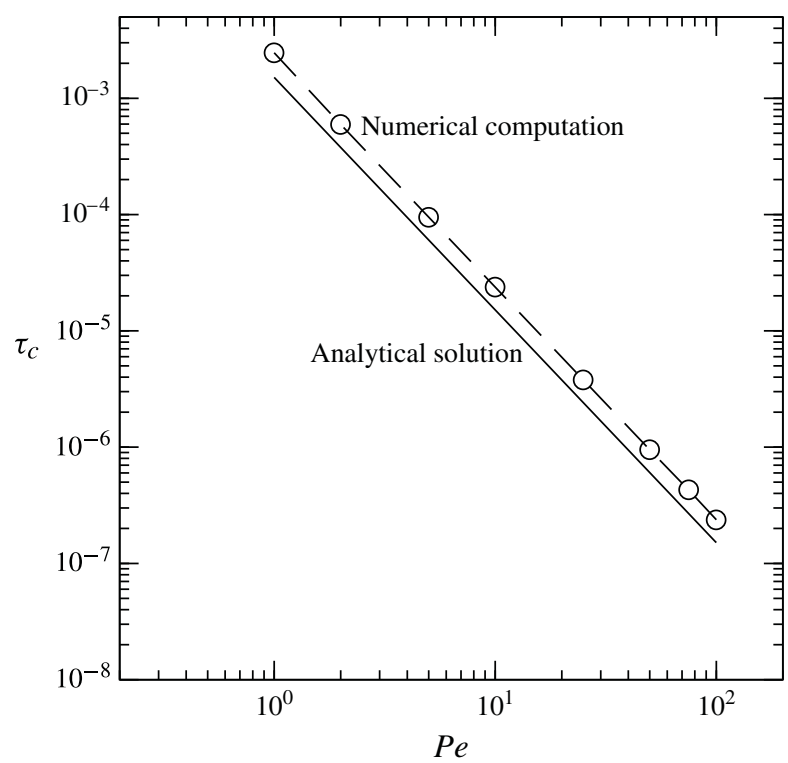

FIGURE 11. Dimensionless time of first precipitation for a uniform evaporation flux at the surface as a function of Péclet number. Comparison between the time obtained from the numerical solution of the dissolved salt transport problem in one dimension and the time given by the analytical prediction (4.18).

Combining the above equation leads to the following results when $C^{\prime}=1$ (onset of crystallization) at the porous medium surface,

$$
\xi_{c}^{\prime}=-P e^{-1} \operatorname{Ln}\left(\frac{C_{c r i s}}{C_{0}}\right)
$$

and

$$
\tau_{c}=P e^{-2}\left(\frac{C_{c r i s}}{C_{0}}-\operatorname{Ln}\left(\frac{C_{c r i s}}{C_{0}}\right)-1\right) .
$$

The prediction given by (4.18) is compared in figure 11 with the prediction obtained from the numerical solution to the original transient problem (4.4)-(4.5) for $C_{0}=25 \%$ and $C_{c r i s}=C_{s a t}=26.4 \%$. As can be seen, the agreement is quite good.

\subsubsection{Numerical solution in cylindrical coordinates}

The 1D formulation used in the previous section cannot predict the peripheral effect visible in figure 9. To capture this effect, the problem governing the dissolved salt mass fraction is solved using cylindrical coordinates. The key ingredient is that the (continuum) evaporation flux distributions depicted in figure 3 are used in (4.11) to compute the velocity field in the porous medium. As already discussed and shown in figure 3, the evaporation flux is greater at the periphery when the distance $\delta$ is sufficiently small.

Since it has been shown that the porosity heterogeneities could have a great impact on the location of efflorescence at the porous surface (Veran-Tissoires et al. 2012b), the impact of the possible different arrangement of the beads near the wall, i.e. the porosity wall effect, is taken into account in the computations through (3.13). 
The results are depicted in figure 12. As can be seen, the peripheral effect is well predicted. The effect is due to the greater evaporation fluxes at the surface periphery (see figure 3), which induce greater velocities in the upper region of the porous medium close to the hollow cylinder wall. Owing to the significance of the advection effect, the higher salt concentrations at the surface are directly correlated with the higher solution velocities in the porous medium periphery. As expected, the porosity increase near the wall (3.13) has a significant impact. The greater porosity near the wall induces a lower interstitial velocity (we recall that the interstitial velocity $\boldsymbol{U}=\boldsymbol{V} / \varepsilon$, where $\boldsymbol{V}$ is the filtration velocity), and therefore a lesser advection effect in the dissolved salt transport. The effect is not sufficient to overcome the peripheral effect due to the increase in the evaporation flux with $r$, i.e. the distance from the surface centre. According to figure 12, it is expected that the first crystals are seen at a distance of approximately one or two bead diameters from the wall. This is not what can be seen from figure 9 where some efflorescence structures are clearly attached to the wall. However, figure 9 corresponds to elapsed times relatively long after the onset of first crystals. Interestingly, the close inspection of the images obtained for the case $\delta=2.5 \mathrm{~mm}$, do indicate that the very first efflorescence structures do not form at the wall but at a distance from the wall consistent with the concentration profile depicted in figure 12. This does not hold, however, for the case $\delta=7.5 \mathrm{~mm}$. No near-wall exclusion distance is observed in the experiment but the peripheral preferential onset is marked, in agreement with the concentration profiles depicted in figure 12. By contrast, the peripheral effect still predicted by the continuum computation for the case $\delta=15 \mathrm{~mm}$ is not seen at all at in the experiment. The first crystal appears actually close to the centre of the surface and not at the periphery.

The continuum approximation is in fact not sufficient to explain the distribution of salt structures shown in figure $9(c)$, nor of course the discrete distributions of precipitation sites also visible in figure $9(a, b)$.

\subsection{Pore network model}

As discussed in $\S 3$, the evaporation flux varies from one surface opening to the other not only because of the peripheral effect but also because of the spatial fluctuations in the sizes of openings. The objective is to take into account this effect in the analysis of the distribution of the salt concentration maxima at the surface of the porous medium. To this end, pore network simulations of the salt transport were developed. As sketched in figure 13, a cubic network was considered. In this type of model (e.g. Blunt et al. 2002; Joekar-Niasar, Hassanizadeh \& Dahle 2010), the pore space is conceptualized as a network of randomly sized pores (located at the nodes of the cubic mesh) joined by randomly sized throats. As sketched in figure 14, each node of network is connected to six neighbour nodes. Note that a node is also located within the top half of each interfacial bond (figure 14b). An interfacial throat at the top surface is a throat oriented along the $z$ direction whose entrance is level with the surface. Thus, the menisci at the surface are located at the entrance of interfacial bonds. Each of these interfacial nodes is therefore connected to only one node. The lattice spacing, i.e. the distance $a$ between two nodes is $d_{p}$, thus corresponding to a bead diameter, except at the surface where the distance is $0.75 d_{p}$ (see figure $14 b$ ).

The pore network simulations are performed as follows. First, a realization of the random pore network geometry is generated (with the parameters detailed previously) and the interface geometry (throat diameter and position) is exported as input geometry for an OpenFoam computation. Hence, the top surface geometry 


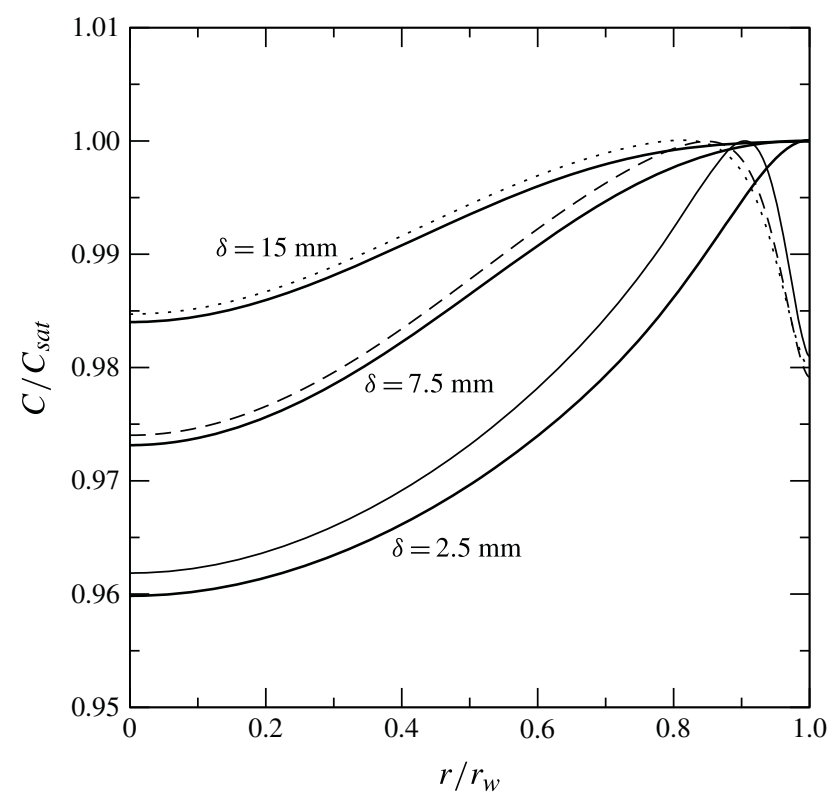

FIGURE 12. Continuum computation of distribution of salt mass fraction at the porous medium surface when the crystallization mass fraction is reached assuming $C_{c r i s}=C_{\text {sat }}$. The thick lines are for a uniform porosity. The thin lines are when the porosity increases near the wall. Note the shift in the position of the mass fraction maximum toward the centre of the surface when the porosity wall effect is taken into account.

is used to create the geometry and mesh for an OpenFoam computation of the evaporation rates at the entrance of each opening at the surface. The OpenFoam computation is similar to those described in $\$ 3.2$. The computed evaporation rate over each opening is then imported as input data for the pore network simulation. The problem governing the pressure in the network problem is then solved and the velocity field inside each bond of the network is computed. Finally, the transient concentration problem is solved until the saturation concentration is reached in an interfacial throat. The details on this pore network model are given in the Appendix.

\subsection{Precipitation onset times, supersaturation effect}

The comparison between the theoretical, numerical and experimental precipitation onset times is presented in table 2 when no supersaturation effect is taken into account in the computations, i.e. $C_{\text {cris }}=C_{\text {sat }}$. Experimentally, the onset time, also referred to as the time of first precipitation, corresponds to the first time that crystals are visible at the surface. Given the limitation/accuracy of the imaging technique used in the experiments, the very first salt crystals (which appear at the nanoscale) cannot be actually visualized. The crystal should, in fact, be greater than about one pixel (which corresponds to a size of $25 \mu \mathrm{m}$ ). A side experiment made with a capillary tube indicates that the growth of sodium chloride crystal up to the size of the capillary diameter is quite rapid. It is thus surmised that the elapsed time between the formation of first crystals and a crystallized structure visible at the surface is quite short. It is therefore concluded that the elapsed time between the moment when $C=C_{c r i s}$ in the experiment and the moment when the first crystallized 


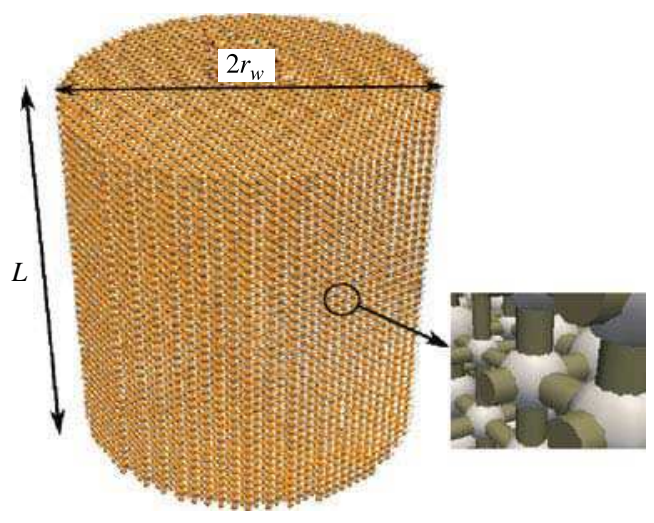

FIGURE 13. (Colour online) Cubic network of interconnected channels as a model porous medium.

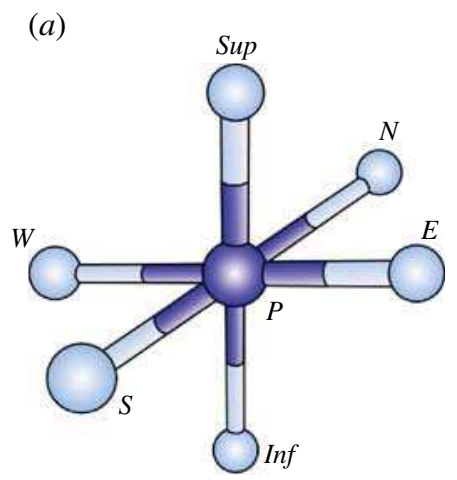

(b)

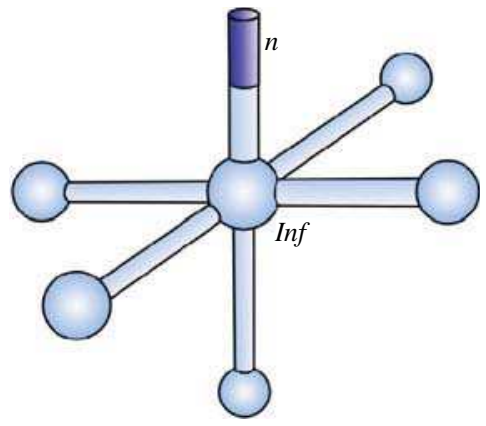

FIGURE 14. (Colour online) (a) Sketch of a pore element in network with its neighbours. (b) Sketch of an interfacial element (throat). The dark region represents the volume associated with the considered element.

structure is visible at the surface must be short. Although this would deserve to be further explored, it is thence concluded that this point cannot be invoked to explain possible discrepancies between the theoretical predictions and the observations. Note also that the image acquisition frequency (one image every $300 \mathrm{~s}$ ) is too low for detecting accurately the precipitation onset time for the case $\delta=2.5 \mathrm{~mm}$. Thus, the experimental time $300 \mathrm{~s}$ indicated in table 2 should be regarded as a conservative estimate.

To perform the 2D continuum simulations, the free convection distributions of the evaporation flux at the surface are used after rescaling so as to obtain the same evaporation rate as in the experiments. A similar procedure is performed for the Péclet number simulations, i.e. a rescaling so as to obtain the same evaporation rate as in the experiment (however, we recall that free convection effects were not considered in the study of evaporation flux from the discrete surface shown in figure 4).

As can be seen from table 2, all numerical approaches underpredict the time of first precipitation except for the case $\delta=2.5 \mathrm{~mm}$, for which the time of first precipitation is quite short. The theoretical approach (4.18) leads to reasonable values compared 


\begin{tabular}{|c|c|c|c|c|c|c|c|c|c|}
\hline Bead size $d_{b}$ & $\delta(\mathrm{mm})$ & $L_{s}(\mathrm{~mm})$ & $P e($ exp.) & Theory equation (4.18) (s) & $\begin{array}{l}2 \mathrm{D} \text { continuum } \\
\varepsilon \text { cte }(\mathrm{s})\end{array}$ & $\begin{array}{l}\text { simulations }(\mathrm{CS}) \\
\qquad \varepsilon(r)(\mathrm{s})\end{array}$ & $\begin{array}{r}P e \text { si } \\
\varepsilon \text { cte }(\mathrm{s}\end{array}$ & $\begin{array}{l}\text { mulations } \\
\qquad \varepsilon(r)(\mathrm{s})\end{array}$ & Experiment \\
\hline $1 \mathrm{~mm}$ & 2.5 & $\approx 10$ & 3 & 437 & 273 & 363 & 460 & 601 & 300 \\
\hline $1 \mathrm{~mm}$ & 7.5 & $\approx 10$ & 2.37 & 561 & 639 & 685 & 741 & 862 & 2700 \\
\hline $1 \mathrm{~mm}$ & 15 & $\approx 10$ & 1.67 & 766 & 1040 & 1082 & 978 & 1125 & 30600 \\
\hline $1 \mathrm{~mm}$ & 15 & 22 & 1.48 & 975 & 1314 & 1375 & 1209 & 1394 & 10800 \\
\hline $100-125 \mu \mathrm{m}$ & 15 & 22 & 1.43 & 1045 & 1405 & 1431 & - & - & 5400 \\
\hline $50-62 \mu \mathrm{m}$ & 15 & 22 & 1.44 & 1030 & 1389 & 1409 & - & - & 4800 \\
\hline $5-50 \mu \mathrm{m}$ & 15 & 22 & 1.57 & 867 & 1177 & 1194 & - & - & 4800 \\
\hline
\end{tabular}

TABle 2. Precipitation onset time assuming $C_{c r i s}=C_{s a t}$ in the simulations. Here $L_{s}$ is the vertical distance between the porous medium surface and the level of the solution in the reservoir (see figure 2). Here $P e=J L / \rho_{\ell} A \varepsilon D_{s}^{*}$ with $\varepsilon=0.363$ and $\rho_{\ell}=1190 \mathrm{~kg} \mathrm{~m}^{-3}$. 
to the $2 \mathrm{D}$ continuum approach and the Péclet number simulations. This was expected for the case $\delta=15 \mathrm{~mm}$ since the evaporation flux is quasi-uniform for this value of $\delta$ (see figure 3) but not for the smaller values of $\delta$ for which the problem is clearly not $1 \mathrm{D}$. The consideration of the porosity wall effect in the $2 \mathrm{D}$ continuum simulations and the Péclet number simulations lead to slightly larger values of the time of first precipitation. This was expected since the increase in porosity near the wall, that is in the region of higher evaporation flux at the surface, leads to local lower interstitial velocities (the interstitial velocity is inversely proportional to the porosity) and, thus, to a lesser advective effect in the near-wall region. Not surprisingly, the Péclet number and continuum simulations lead to similar results, which was of course expected from the construction of the Péclet number model (see the Appendix). Note that only one realization of network is considered throughout the present study. However, the consideration of several realizations does not change the main results (Veran-Tissoires 2011).

The time of first precipitation decreases with the Péclet number as expected. Interestingly, the experiments with the various sizes of beads lead to comparable times of first precipitation, except for the $1 \mathrm{~mm}$ beads, with a time about twice as long as for other diameters. This difference is not captured by the simulations. The experimental results also indicate that the level in the reservoir (distance $L_{s}$, see figure 2) is a sensitive parameter. The comparison between the experiment for $L_{s}=22 \mathrm{~mm}$ and that for the lower $L_{s}(\approx 1 \mathrm{~cm})$ leads to a factor of three in the time of first precipitation $(\delta=15 \mathrm{~mm})$. This would indicate that the exact position of menisci between the beads at the surface has some impact, at least for sufficiently large beads.

The fact that the simulations lead to lower precipitation times (with the only exception of the case $\delta=2.5 \mathrm{~mm}$ which is characterized by a quite short precipitation time) is interpreted as a supersaturation effect. To evaluate the supersaturation we have performed for each case the simulations over a longer period, the period corresponding to the experimental time of first precipitation as reported in table 2 . This leads to crystallization salt mass fractions greater than the solubility mass fraction and thus to supersaturation. The results are summarized in table 3. Except again for the case $\delta=2.5 \mathrm{~mm}$, which leads to a time of first precipitation at least one order of magnitude smaller than in the other cases and no supersaturation effect, this procedure leads to supersaturations in the range (1.05-1.26). This confirms the existence of a noticeable supersaturation effect. According to the results reported in table 3, the supersaturation does not seem to depend greatly on the bead diameter, which is in agreement with experiments with capillary tubes of different diameters (Desarnaud et al. 2014).

\subsection{Locations of salt concentration maxima at the surface}

The key aspect discussed in this section is that the ion concentration at the surface is not locally uniform, i.e. varies from one meniscus to another. This non-uniformity is distinct from the non-uniformity associated with the peripheral effect (greater evaporation flux at the periphery) or the wall porosity effect. The heterogeneous nature of the ion distribution discussed here is a direct consequence of two factors: (i) the significance of the advection effect in the transport of ions toward the evaporative surface; (ii) the disordered nature of the porous medium at the pore network scale. As a result of the spatial variations in the pore size throughout the pore space, the velocity field at the pore network scale is not uniform: the average 


\begin{tabular}{|c|c|c|c|c|c|c|c|}
\hline Bead size $d_{b}$ & $\delta(\mathrm{mm})$ & $L_{s}(\mathrm{~mm})$ & Time of first precipitation (Exp.) (s) & $\mathrm{Pe}$ & \multicolumn{3}{|c|}{ 2D continuum simulations } \\
\hline $1 \mathrm{~mm}$ & 2.5 & $\approx 10$ & 300 & 3 & $\begin{array}{c}C_{c r i s} \\
C_{c r i s} / C_{s a t}\end{array}$ & $\begin{array}{c}0.2648 \\
1.003\end{array}$ & $\begin{array}{c}0.2628 \\
0.995\end{array}$ \\
\hline $1 \mathrm{~mm}$ & 7.5 & $\approx 10$ & 2700 & 2.37 & $\begin{array}{c}C_{c r i s} \\
C_{c r i s} / C_{s a t}\end{array}$ & $\begin{array}{c}0.2795 \\
1.06\end{array}$ & $\begin{array}{c}0.2779 \\
1.05\end{array}$ \\
\hline $1 \mathrm{~mm}$ & 15 & $\approx 10$ & 30600 & 1.67 & $\begin{array}{c}C_{c r i s} \\
C_{c r i s} / C_{s a t}\end{array}$ & $\begin{array}{c}0.3319 \\
1.26\end{array}$ & $\begin{array}{c}0.3265 \\
1.26\end{array}$ \\
\hline $1 \mathrm{~mm}$ & 15 & 22 & 10800 & 1.48 & $\begin{array}{c}C_{c r i s} \\
C_{c r i s} / C_{s a t}\end{array}$ & $\begin{array}{c}0.2915 \\
1.1\end{array}$ & $\begin{array}{c}0.2895 \\
1.1\end{array}$ \\
\hline $100-125 \mu \mathrm{m}$ & 15 & 22 & 5400 & 1.43 & $\begin{array}{c}C_{c r i s} \\
C_{c r i s} / C_{s a t}\end{array}$ & $\begin{array}{c}0.2780 \\
1.05\end{array}$ & $\begin{array}{c}0.2776 \\
1.05\end{array}$ \\
\hline $50-62 \mu \mathrm{m}$ & 15 & 22 & 4800 & 1.44 & $\begin{array}{c}C_{c r i s} \\
C_{c r i s} / C_{s a t}\end{array}$ & $\begin{array}{c}0.2765 \\
1.05\end{array}$ & $\begin{array}{c}0.2763 \\
1.05\end{array}$ \\
\hline $5-50 \mu \mathrm{m}$ & 15 & 22 & 4800 & 1.57 & $\begin{array}{c}C_{c r i s} \\
C_{c r i s} / C_{s a t}\end{array}$ & $\begin{array}{c}0.2788 \\
1.06\end{array}$ & $\begin{array}{c}0.2787 \\
1.06\end{array}$ \\
\hline
\end{tabular}

TABLE 3. Degree of supersaturation to obtain a perfect agreement between the simulations and the experiment on the time of first precipitation. Here $L_{s}$ is the vertical distance between the porous medium surface and the level of the solution in the reservoir (see figure 2). 
velocity in the passages between two pores fluctuates spatially. It is well known that the transport of a species in a stationary random velocity field leads to the phenomenon of dispersion (e.g. Koch \& Brady 1985 and references therein). Here we have a similar effect, spatial fluctuations in the ion concentration at the pore network scale induced by the spatial fluctuations of the velocity field. Note however that the Péclet number based on a pore scale classically considered in the studies on dispersion in porous media is lower than one in our experiments (the Péclet number considered in the previous sections is based on a macroscopic scale, the height of the sample, not on a characteristic length at the pore scale). This explains why the coefficient $D_{s}^{*}$ in (4.1) is a diffusion coefficient and not a dispersion coefficient. Interestingly, although the pore-scale Péclet number is small, the fluctuations in the velocity field have nevertheless a significant impact on the ion distribution. Two sources of velocity fluctuations can be distinguished. The first is the fluctuations induced by the internal disorder of the porous medium. By analogy with classical dispersion in porous media, this internal disorder effect could be called the 'dispersion' effect even if the pore scale Péclet is much smaller than one as discussed previously. We decided to name this the 'internal disorder effect'. The second is due to the local fluctuations in the evaporation flux at the surface as discussed in $\S 3.2$, which in turn induce fluctuations in the average velocity in the interfacial throats. This effect is referred to as the 'surface disorder effect'.

The influence of both effects is discussed from pore network simulations in what follows.

We first discussed the ability of pore network model to capture the localisation of maxima at the surface. The simulation is stopped when $C=C_{c r i s}$ somewhere at the surface. As mentioned before, there are approximately 1000 points at the surface where the ion concentration is computed with the pore network model. Ranking the nodes at the surface from that of highest concentration $\left(=C_{\text {cris }}\right)$ to that of lower concentration, we have decided to concentrate on the 20 first points in this list, that is on the 20 first points of highest concentrations at the surface. The results are depicted in figure 9. For each value of $\delta$, two simulations were performed. The full simulations (figure $9 d-f$ ) take into account all effects, including the increase in the porosity near the wall (3.13). The latter is neglected in the second series of simulations (figure $9 g-i$ ). As can be seen the discrete peripheral effect is well captured by the pore network simulations for the cases $\delta=2.5 \mathrm{~mm}$ and $\delta=7.5 \mathrm{~mm}$ in accordance with the experiments. The simulations without the porosity wall effect lead to maxima located much closer to the edge of the surface than with the porosity wall effects. As discussed previously, the simulations including the porosity wall effects are in better agreement with the observations of the films showing the formation of crystals at the surface of porous medium. This is of course much more obvious for the case $\delta=15 \mathrm{~mm}$. When the porosity wall effect is neglected, the slightly greater evaporation flux at the periphery (figure 3 ) is sufficient to lead to the preferential onset of precipitation at the periphery (figure 9i). Thus, the concentration fluctuations induced by the internal disorder and the surface disorder are not sufficient to overcome the effect of the slightly greater evaporation flux at the periphery. By contrast, the results of pore network simulation are much more consistent with experiments when the porosity wall effect is taken into account (compare figure $9 c$ with figure $9 f, i)$. In summary, the pore network simulations fully support the physical interpretation that the discrete precipitation spots seen in the experiments originate from the ion concentration fluctuations resulting from the transport of the ions in a velocity field heterogeneous at the pore network scale. All of the factors affecting 
(a)

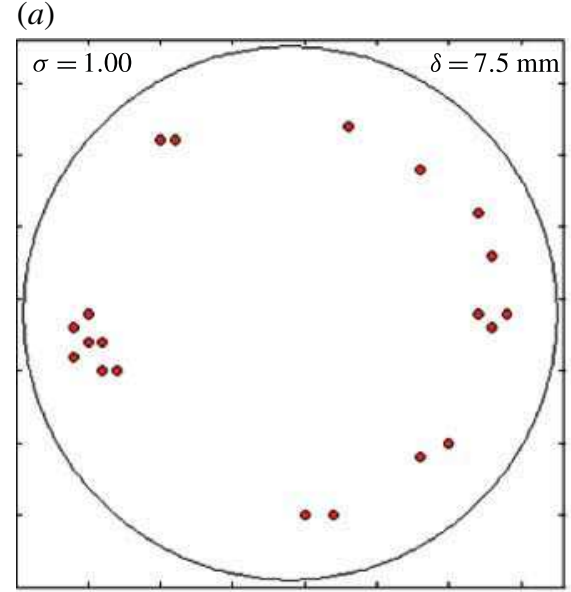

(c)

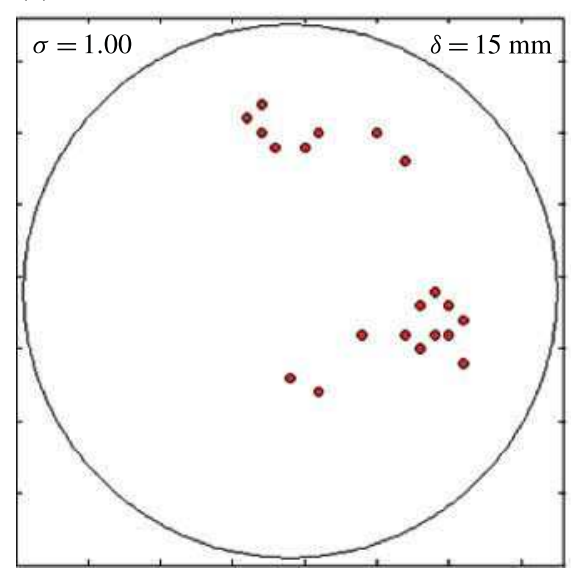

(b)

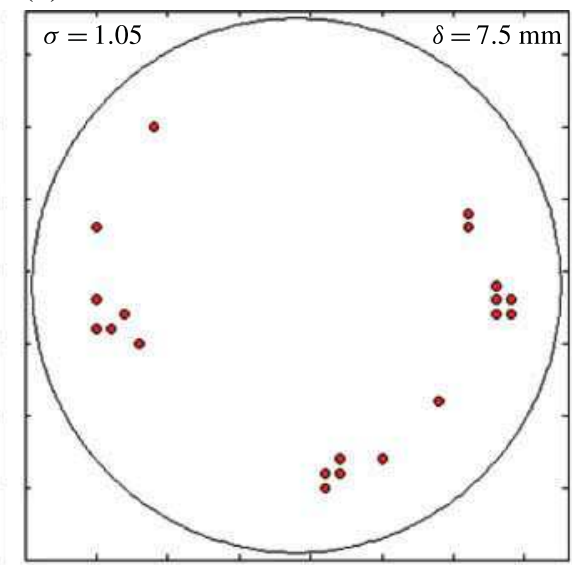

(d)

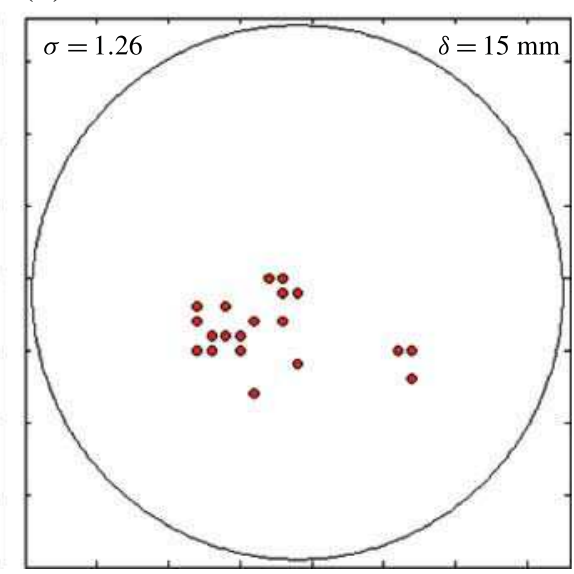

FIGURE 15. (Colour online) Influence of supersaturation $\sigma$ on the position of twenty greater salt concentration maxima at the surface of the porous medium from pore network simulations. The supersaturations imposed for the computations when $\sigma>1$ (column on the right) correspond to the values reported in table 3 .

the velocity spatial variations and fluctuations should therefore be taken into account to predict the distribution of precipitation onset points at the surface.

The Péclet number simulation results reported in figure 9 were obtained assuming a negligible supersaturation $\left(C_{c r i s} \approx C_{\text {sat }}\right)$. Taking into account the non-negligible supersaturations determined in $\S 4.4$ and presented in table 3 leads to the results shown in figure 15. As can be seen, assuming a greater supersaturation does not change qualitatively the results but interestingly indicates that the position of maxima at the surface changes with time.

This point is further investigated hereafter neglecting the porosity wall effect for the case $\delta=15 \mathrm{~mm}$. Figure 16 shows the evolution of ion mass fraction in the interfacial throats at different times up to steady state (we have thus run the simulation up to steady state no matter the level of the ion mass fraction at the surface). To characterize the evolution of salt concentration maxima at the surface, we computed the following 
correlation coefficients,

$$
\begin{gathered}
\chi(t)=\frac{\sum_{1}^{n}\left(C_{i}(t)-\bar{C}(t)\right)\left(C_{i}\left(t_{f}\right)-\bar{C}\left(t_{f}\right)\right)}{\sqrt{\sum_{1}^{n}\left(C_{i}(t)-\bar{C}(t)\right)^{2}} \sqrt{\sum_{1}^{n}\left(C_{i}\left(t_{f}\right)-\bar{C}\left(t_{f}\right)\right)^{2}}} \\
\omega(t)=\frac{\sum_{1}^{n}\left(C_{i}(t)-\bar{C}(t)\right)\left(u_{i}(t)-\bar{u}(t)\right)}{\sqrt{\sum_{1}^{n}\left(C_{i}(t)-\bar{C}(t)\right)^{2}} \sqrt{\sum_{1}^{n}\left(u_{i}(t)-\bar{u}(t)\right)^{2}}}
\end{gathered}
$$

where $\chi(t)$ is the correlation coefficient between the distribution of $C$ at time $t$ and the steady-state distribution $C\left(t_{f}\right)$ whereas $\omega(t)$ is the correlation coefficient between the distribution of $C$ at time $t$ and the distribution of the velocity $u$ in the interfacial bonds of pore network, i.e. with the distribution of evaporation flux $j_{o}$ from the menisci located at the entrance of interfacial bonds since $u=j_{o} / \rho_{\ell}$.

As can be seen from figure 16, the position of the maxima is dominated by the local fluctuations in the evaporation flux at the surface, i.e. by the surface disorder, at short times. The ion concentration maxima correspond to the interfacial throats of higher evaporation flux ( $=$ of higher induced average velocity). Then the correlation between the local evaporation fluxes and the salt concentration peaks dies out as the salt concentration gradient zone expands from the surface (figure 10). As reported in table 2 , the Péclet number is of the order of 1.5 , which gives $\xi\left(t_{f}\right) \approx L / P e \approx 24$ bead diameters. Thus the ion concentration gradient zone when the steady state is reached is sufficiently wide for the impact of internal disorder to greatly affect the distribution of salt concentration peaks at the surface. In brief, the surface disorder is dominant at short times whereas the internal disorder is dominant at longer times provided that $\xi\left(t_{f}\right)$ is not too small, i.e. $\xi\left(t_{f}\right)$ is greater than a few bead diameters. This is confirmed by the comparison between figures 16 and 17, which shows that $\chi(t)$ decreases sharply as soon as $\xi(t)$ increases.

In summary, the simulations indicate that the distribution of concentration peaks at the porous medium surface is not frozen. There is a permanent reorganization of the maxima distribution with time. This can be related in part to the dynamic evolution of the crystallized salt structures discussed in $\S 5$. The dynamic evolution revealed by figures 15 and 16 results from the effect of the internal disorder effect (dispersion). As noted before, the size $\xi(t)$ of the concentration gradient zone adjacent to the surface (see figure 10) increases with time. Thus, the region where the ion distribution is impacted by the internal disorder increases in time and this affect the ion distribution at the interface.

\section{On the efflorescence growth}

\subsection{Patchy efflorescence is porous}

As discussed in previous papers (Sghaier \& Prat 2009; Veran-Tissoires et al. 2012a,b), a patchy efflorescence is porous. This can be illustrated from a simple impregnation (Washburn) experiment. A dry efflorescence structure is gently detached from the 


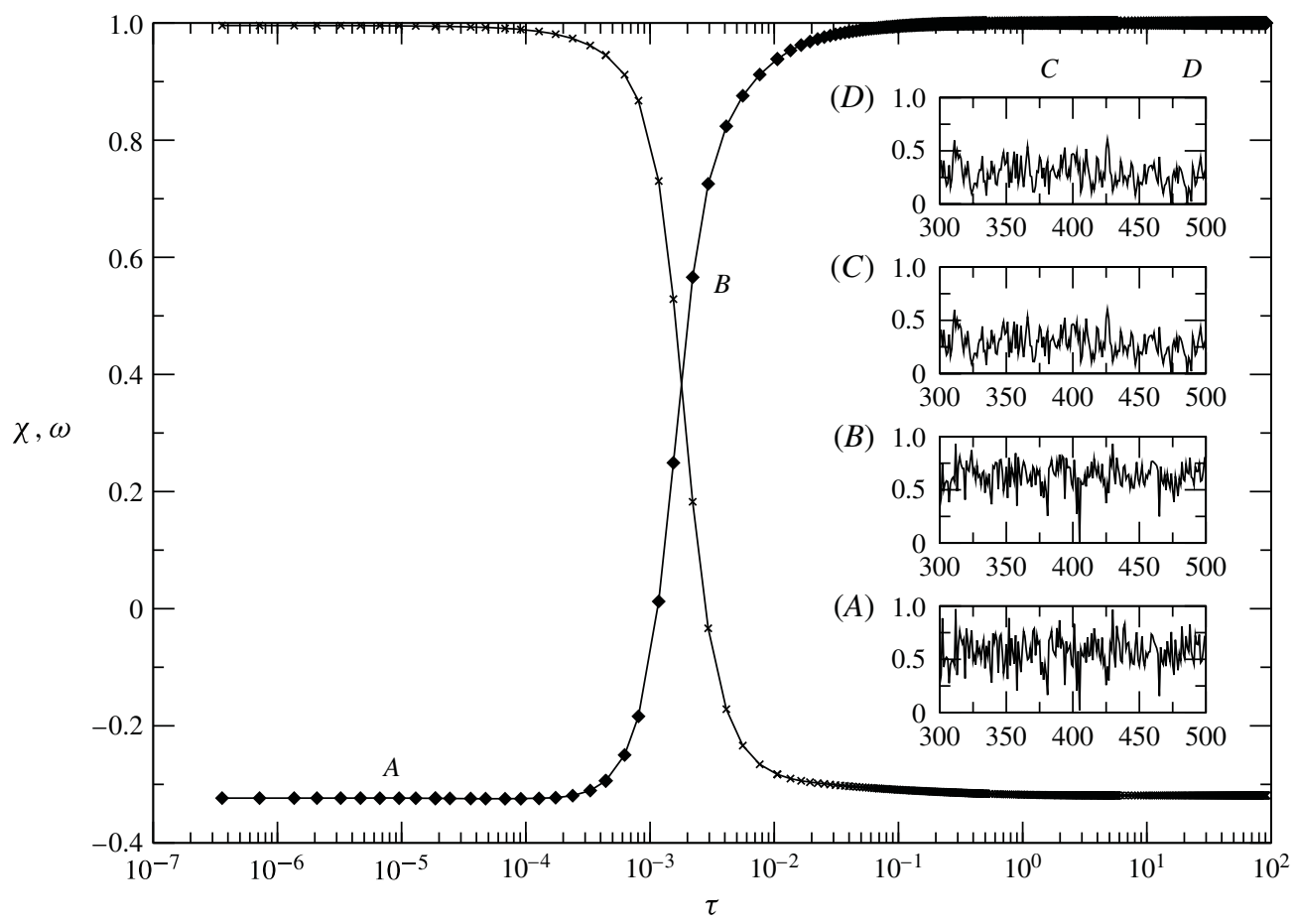

FIGURE 16. Black line with diamonds: evolution of correlation coefficient $\chi(t)=$ $\left(\sum_{1}^{n}\left(C_{i}(t)-\bar{C}(t)\right)\left(C_{i}\left(t_{f}\right)-\bar{C}\left(t_{f}\right)\right)\right) /\left(\sqrt{\sum_{1}^{n}\left(C_{i}(t)-\bar{C}(t)\right)^{2}} \sqrt{\sum_{1}^{n}\left(C_{i}\left(t_{f}\right)-\bar{C}\left(t_{f}\right)\right)^{2}}\right)$ between salt mass fraction distribution at the pore network surface at time $t$ and ion mass fraction distribution at the pore network surface corresponding to the steady state distribution. Black line with $\mathrm{x}$ : evolution of correlation coefficient $\omega(t)=\left(\sum_{1}^{n}\left(C_{i}(t)-\bar{C}(t)\right)\left(u_{i}(t)-\right.\right.$ $\bar{u}(t))) /\left(\sqrt{\sum_{1}^{n}\left(C_{i}(t)-\bar{C}(t)\right)^{2}} \sqrt{\sum_{1}^{n}\left(u_{i}(t)-\bar{u}(t)\right)^{2}}\right)$ between ion mass fraction distribution at the surface and the interstitial velocity in the interfacial throats at time $t$. The insets show the normalized ion mass fraction distribution at the surface at different times. The normalized ion mass fraction is $C_{i}^{*}(t)=\left(C_{i}(t)-C_{\min }(t)\right) /\left(C_{\max }(t)-C_{\min }(t)\right)$ where $C_{\max }$ and $C_{\min }$ are the maximum and minimum ion mass fractions at the surface at time $t$, respectively.

porous surface. The structure is then hung on a scale and put in contact at its base with a red dye ricin oil. Owing to capillary action, the solution is sucked into the efflorescence structure and the mass of the efflorescence increases with time. An example of a result obtained in this spontaneous imbibition experiment is shown in figure 18 for an efflorescence structure of approximately $1 \mathrm{~cm}$ in height. This clearly demonstrates the capillary nature of the efflorescence structure. As shown in the inset, the variation of the mass as a function of time obeys a power law over most of the imbibition dynamics as in a classical (Washburn) imbibition experiment. However, the exponent $(\sim 0.44)$ is lower than the classical exponent 0.5 which is not surprising owing to the shape of the efflorescence and its probably not homogeneous microstructure. 


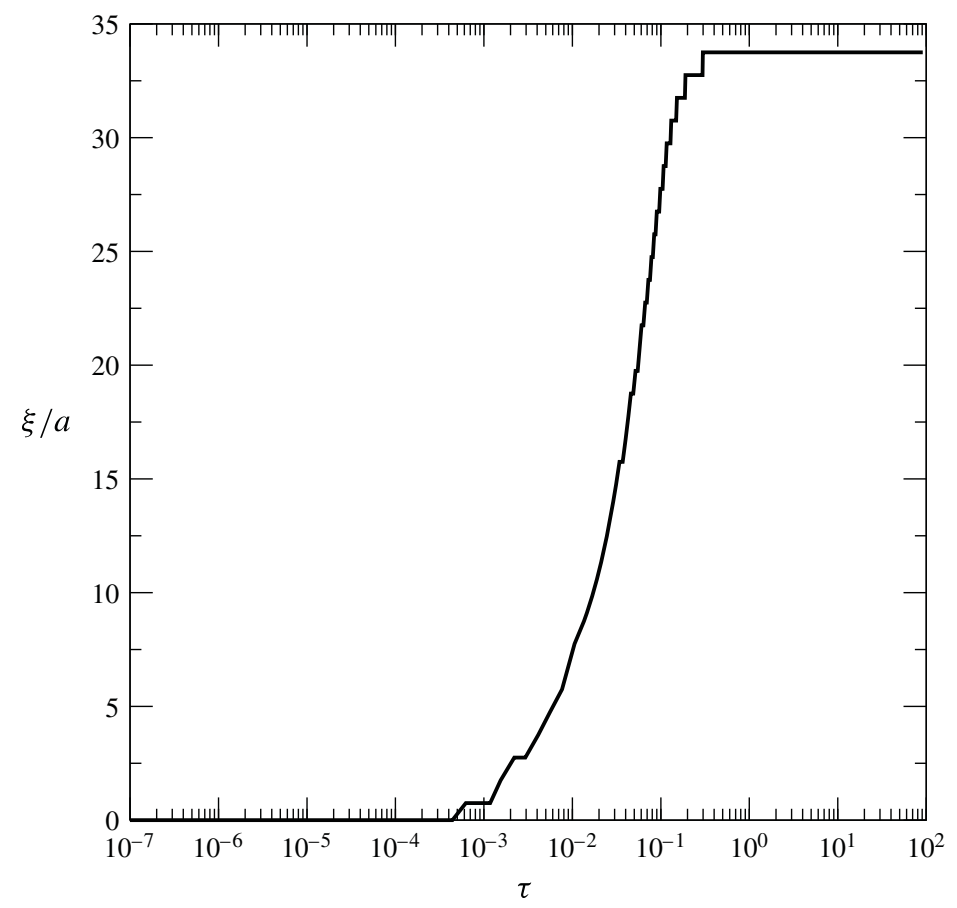

FIGURE 17. Evolution of size $\xi(t)$ of the ion mass fraction gradient zone (see figure 10) with time. Here, $\xi(t)$ is defined as the thickness of the upper layer of the pore network where the mean (= horizontal slice averaged) ion mass fraction is equal or greater than $1.01 C_{0}$ (= at least $1 \%$ greater than $\left.C_{0}\right)$, where $C_{0}$ is the initial ion mass fraction and the ion mass fraction imposed at the bottom of pore network.

\subsection{Patchy efflorescence growth}

The porous nature of an efflorescence structure is important to understand its growth. The ions are in fact transported as the result of the capillary action through the efflorescence structure up to its top where the evaporation flux is higher. As a result the efflorescence structure preferentially grows at its top since crystallization takes place preferentially in the regions of high evaporation flux. Hence, as discussed by Veran-Tissoires et al. $(2012 a, b)$, the porous nature of the patchy efflorescence also explains why the efflorescence continues to grow forming isolated structures leaving surface pores between efflorescence structures free of efflorescence. The efflorescence structures first act as sinks at the porous surface (the dissolved salt is directed toward the efflorescence). The second effect is a screening effect, i.e. the fact that the evaporation flux becomes negligible in the porous surface regions located between the efflorescence structures when the structures have sufficiently grown. Since the velocity in the interfacial throat is proportional to the evaporation flux at the entrance of the throat $\left(u_{i}=j / \rho_{\ell}\right)$, this velocity tends to zero as the nearby efflorescence structures grow. The advection effect is therefore killed and this explains why the crystallization concentration is not reached in the regions of surface located between the growing efflorescence. However, the situation can be slightly subtler than described by Veran-Tissoires et al. (2012a). For instance, it is possible that crystal forms between two growing efflorescence already in place, then stop growing and disappear later when the nearby taller efflorescence structures have sufficiently grown. 


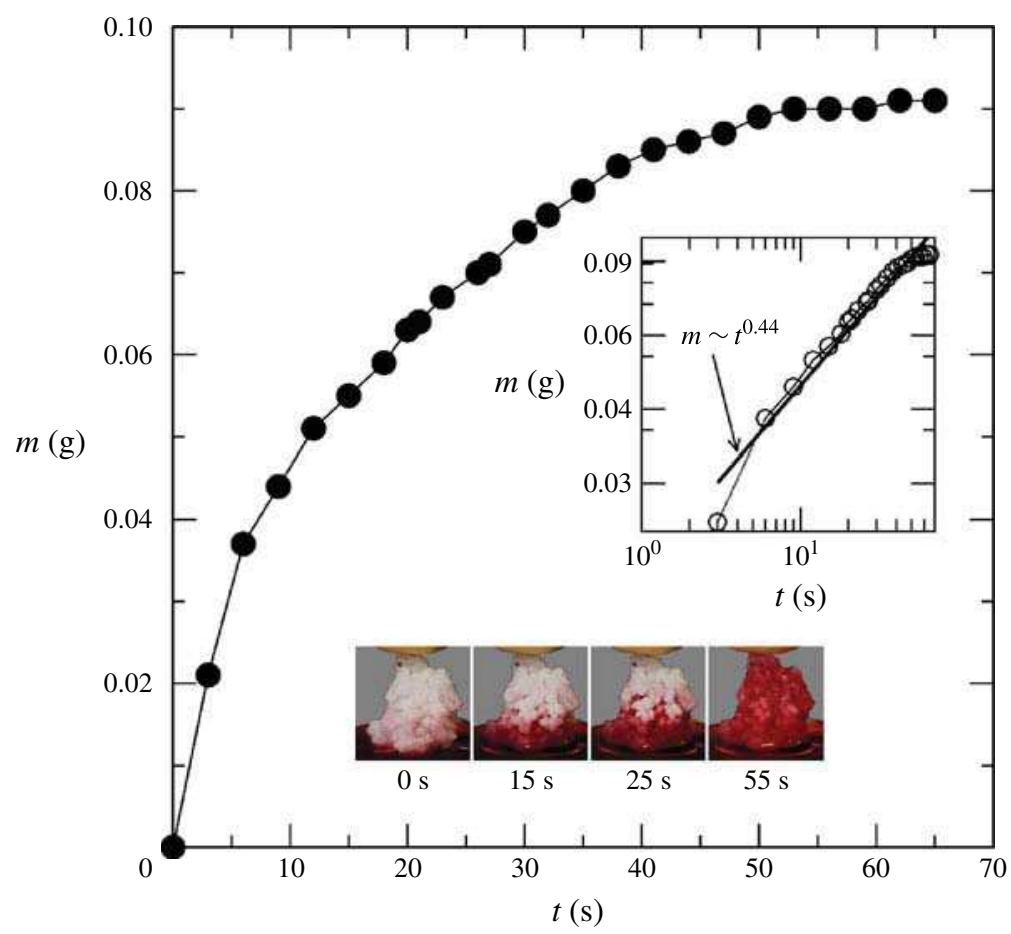

FIGURE 18. (Colour online) Example of impregnation experiment of an initially dry efflorescence structure. Mass of liquid sucked into the efflorescence as a function of time. The inset bottom shows images of the efflorescence structure at different stages of impregnation. The inset top shows the results in $\log -\log$ coordinates.

This is illustrated in figure 19. Figure 19 thus confirms the analysis proposed in Veran-Tissoires et al. (2012a).

\subsection{Dynamic aspect of efflorescence growth: the efflorescence dance}

The images of patchy efflorescence in figure 9 present a static view of efflorescence structures at the surface. In fact, watching the film made from the successive snapshots of the porous medium surface in accelerated mode reveals that the efflorescence growth is a dynamic process characterized by major reorganization of the efflorescence structures. The impression is an efflorescence dance characterized by displacements of the individual efflorescence structures over the surface of the porous medium. The detailed analysis of this dynamics is beyond the scope of the present paper. We have simply selected two results illustrating in part the observed dynamics. Figure 20 shows the evolution of the number of individualized salt structures at the surface as a function of time. As can be seen, the number of efflorescence structures at the surface is not a monotonous function of time. The salt structure population varies because of births (growth of new salt structures), marriages (coalescence of two salt structures) and deaths (a salt structure can disappear as already shown in §5.2). The results reported in figure 20 raise several interesting questions deserving future studies, such as the understanding of the influence of $\delta$ or the evaporation rate on the number of precipitation spots. The dynamic aspect of the efflorescence growth is also illustrated in figure 21 which shows a zoom of the porous medium surface with a cycle of 
(a)

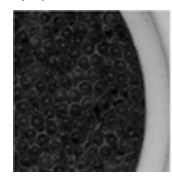

(b)

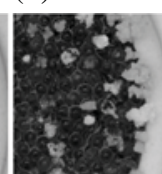

(c)

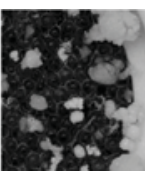

$(d)$

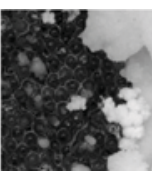

$(e)$

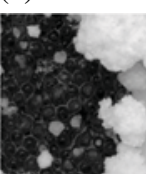

(f)

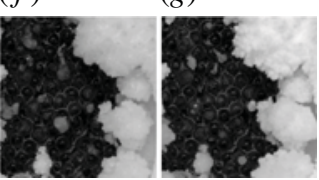

FIGURE 19. Illustration of the efflorescence development as a precipitation-dissolution process: (a) porous medium surface free of efflorescence; $(b, c)$ growth of efflorescence; $(d-g)$ growth of large efflorescence (on the right-hand side of images, close to the wall) and dissolution of small efflorescence structures (in the centre of images).

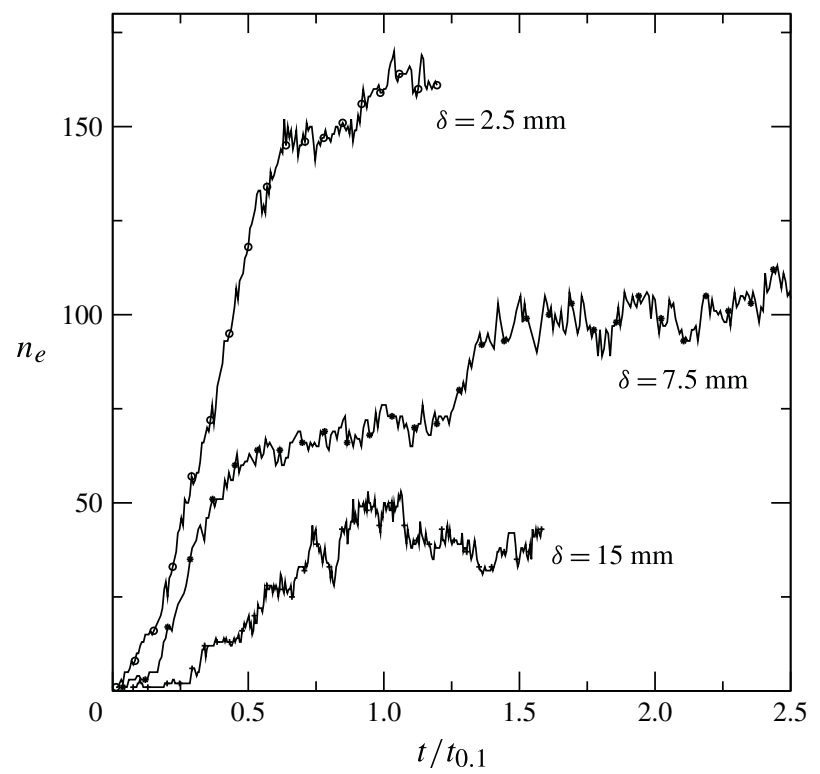

FIGURE 20. Number of individual efflorescence structures as a function of time at the surface of the porous medium. The reference time $t_{0.1}$ corresponds to the time when the fraction of porous medium surface covered by efflorescence on the $2 \mathrm{D}$ images is 0.1 .

growth and dissolution of a salt structure. The observation of the efflorescence dance also indicates possible changes in the colour of a salt structure. The colour change indicates a change in the liquid content within the salt structure. A dry salt structure is much whiter than an imbibed salt structure, which appears greyer when wet. This is visible in figure 21.

This confirms in a more acute way the complex interactions existing between the transfers within the porous medium, the transfers (with precipitation) within the salt structures and the transfers of the vapour in the external air. The coupling between those transfers leads to dynamic, albeit slow, changes in the ion concentration distribution in both the region of the porous medium adjacent to the surface bearing the efflorescence and the salt structures. The main conclusion of this section is therefore that the salt structure development at the surface should not be considered as a 'simple' precipitation process but as a precipitation-dissolution process, in which the precipitation overcomes the dissolution. The existence of the dissolution process is qualitatively explained by the variations of the ion mass fraction field during the 
(a)

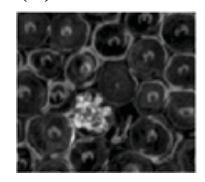

(b)

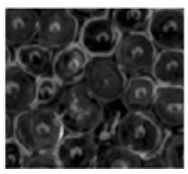

(c)

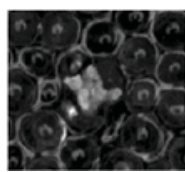

$(d)$

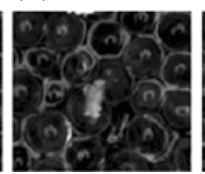

(e)

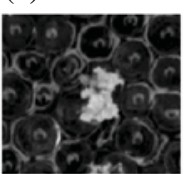

(f)

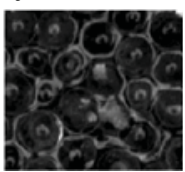

FIGURE 21. Growth and dissolution cycles of efflorescence at the surface of a packing of $1 \mathrm{~mm}$ beads: (a) $660 \mathrm{mn}$; (b) $675 \mathrm{mn}$; (c) $1305 \mathrm{mn}$; (d) $1830 \mathrm{mn}$; (e) $2220 \mathrm{mn}$; (f) $2370 \mathrm{mn}$.

development of efflorescence. In brief the ion mass fraction can decrease and becomes lower than the solubility mass fraction in regions which are not exposed to high evaporation flux anymore because of, for instance, the faster growth of neighbouring efflorescence structures and because of the salt transfer between the solution and the efflorescence structures (the solution can, thus, become less concentrated in some places). All of these need further studies to be explored in detail.

\section{Crusty versus patchy efflorescence}

\subsection{Experiment}

In this section, the distance $\delta$ is set to $\delta=15 \mathrm{~mm}$ and we look at the influence of bead size $d_{b}$ on the interplay between the efflorescence development and the evaporation rate. Four ranges of bead size are considered, namely $(5-50 \mu \mathrm{m}),(50-62 \mu \mathrm{m})$, $(100-120 \mu \mathrm{m})$ and $(1-1.125 \mathrm{~mm})$. This is the only parameter we vary. All other parameters (temperature, $\delta, R H$, etc.) are identical in the experiments discussed in this section. The distance $L_{s}$ between the level of the free surface in the solution reservoir and the porous medium surface at the beginning of each experiment is $22 \mathrm{~mm}$ as mentioned in $\S 2$.

As can be seen from figure 22, two main situations can be distinguished depending on bead size. For sufficiently fine porous medium, the efflorescence eventually forms a crust covering all of the porous medium surface whereas for a sufficiently coarse porous medium the it forms well-individualized structures with a significant fraction of the porous medium surface remaining free of efflorescence. The first type of efflorescence is referred to as 'crusty' and is shown in figure $22(b, d)$. The second type, which was the only one considered in the previous sections, is referred to as 'patchy' and shown in figure $22(f, h)$.

Yet it is clear from figure 22 that the situation is subtler and certainly would require to distinguish more than only two types of efflorescence. Note, for instance, the tessellation pattern obtained with the beads in the range $(5-50 \mu \mathrm{m})$. This pattern looks very much like a small-scale replica of the polygonal patterns often observed at the surface of natural systems, like the Salar de Uyuni in Bolivia for example (e.g. Svendsen 2003). This pattern is quite different from the sort of frozen sea obtained with the beads in the range $(50-62 \mu \mathrm{m})$. Explaining this difference and the mechanisms leading to these different crusty patterns is only one of the numerous questions not yet solved in the field. In passing, we note that one could probably be tempted to think that this tessellation pattern has to do with hydrodynamic instability effects since there is a resemblance with Bénard-Marangoni convection patterns. Free convection effects do exist in the gas phase but there are very limited, the transport in the gas phase being dominated by vapour diffusion. The density differences in 

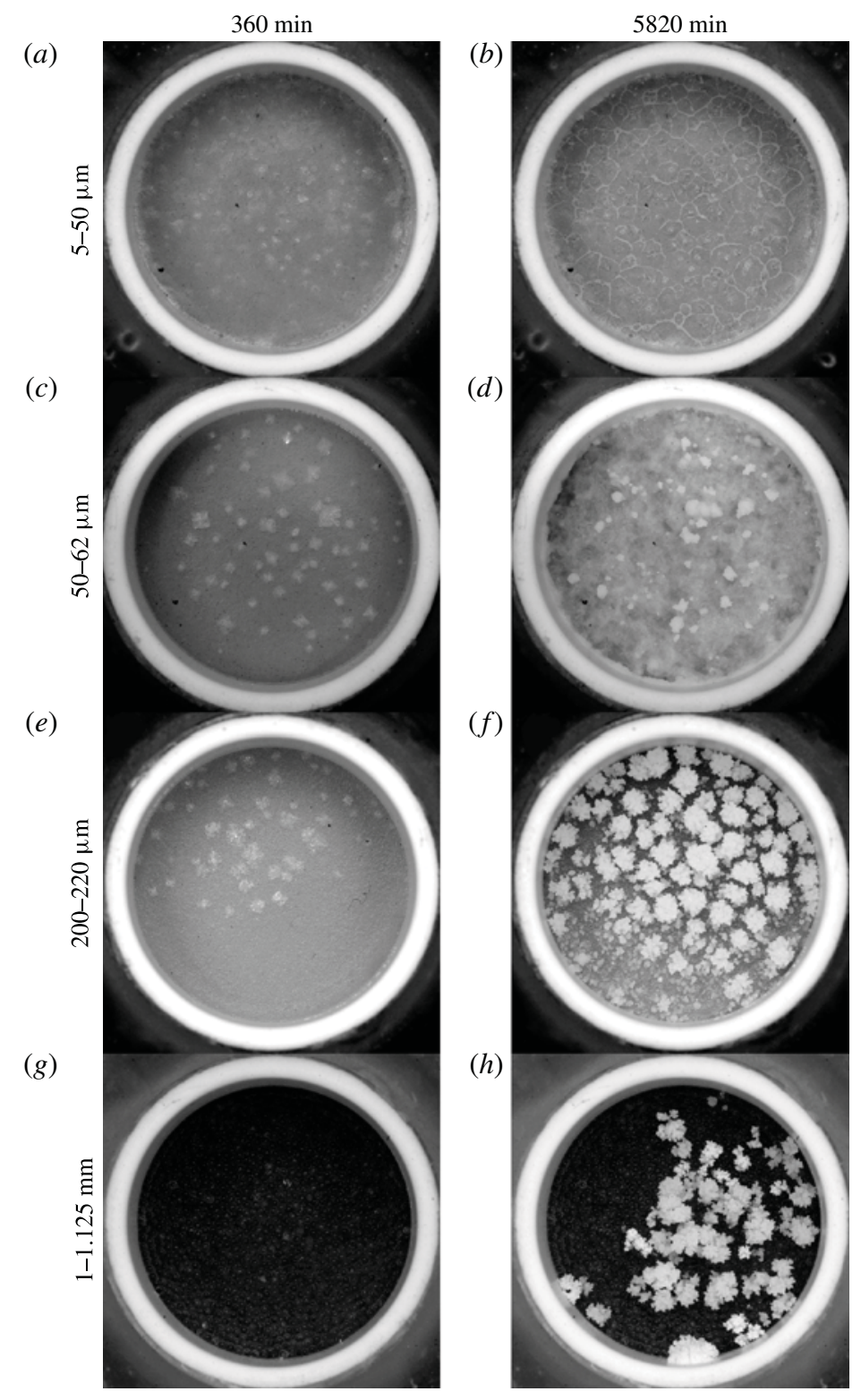

FIGURE 22. Efflorescence patterns obtained by changing the size of the beads forming the porous medium.

the liquid are very small since the salt concentration variations, albeit important to understand the beginning of the efflorescence formation, are small in our experiments (we recall that the solution in the reservoir feeding the porous medium is nearly saturated). Thus, we believe that this puzzling tessellation pattern has nothing to do with free convection effects, see also Svendsen (2003). Figure 23 shows the evolution of the evaporated mass of liquid, $m_{e}$, for the different bead sizes together with the evolution for pure water as well as the evolution obtained by correcting the activity. The water activity for a saturated sodium chloride aqueous solution 


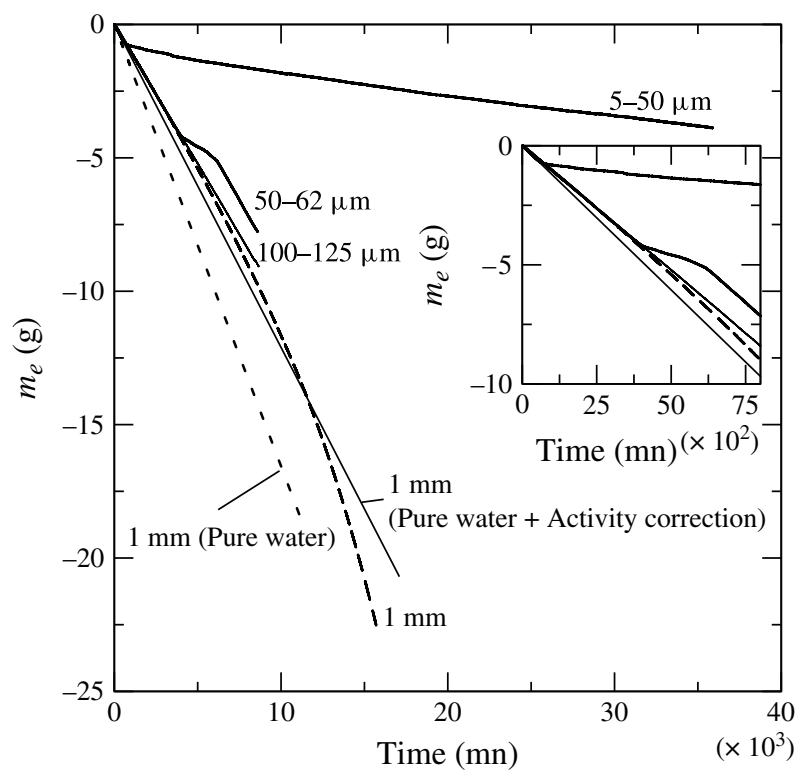

FIGURE 23. Evolution of evaporated mass as a function of time for the various bead sizes tested. The inset shows a zoom of the main figure over the range of time $(0-7500 \mathrm{mn})$.

is $0.75 a_{w 0}$, where $a_{w 0}$ is the activity of pure water. Since the evaporation process is driven by the vapour partial pressure difference $P_{v p m}-P_{v L i b r}$ between the porous medium surface and the $\mathrm{LiBr}$ aqueous solution surface (see figure 2), the evaporation rate of a saturated sodium chloride solution if no crystallization would occur should be $J \approx J_{\text {water }}(0.75-0.07) /(1.0-0.07)$, where $J_{\text {water }}$ is the evaporation rate for pure water and $7 \%$ is the relative humidity at the surface of the $\mathrm{LiBr}$ solution. This correction leads to the straight line labelled 'pure water + activity correction' in figure 23.

Several interesting results can be seen from figure 23. First the type of efflorescence can have a dramatic effect on the evaporation rate. The crust obtained with the $(5-50 \mu \mathrm{m})$ beads severely reduces the evaporation. By contrast, such an effect is not observed for a sufficiently coarse porous medium when the efflorescence is patchy. For example, with the $1 \mathrm{~mm}$ beads, the evaporation rate is in a first phase on the same order of magnitude as the rate that would be obtained with a liquid solution having the same activity as a saturated sodium chloride solution. As can be seen in figure 23 for the experiment with the $1 \mathrm{~mm}$ beads, the development of a patchy efflorescence then leads to evaporation rates even greater than for pure water. This counterintuitive result, also observed in drying experiments as reported in Sghaier \& Prat (2009) and Eloukabi et al. (2013), is explained by the enhanced exchange surface resulting from the development of efflorescence. This effect is sufficient to overcompensate the reduction in the water activity due to the ions.

The evolution reported in figure 23 for the beads in the range $(50-62 \mu \mathrm{m})$ is also instructive. Three main phases can be identified. A first phase of rapid evaporation, slightly slower than for the case of the $1 \mathrm{~mm}$ beads but comparable, is followed by a period during which the evaporation rate decreases quite significantly. This period lasts approximately $25 \mathrm{~h}$. Then the evaporation rate increases and recovers a value 
(a)

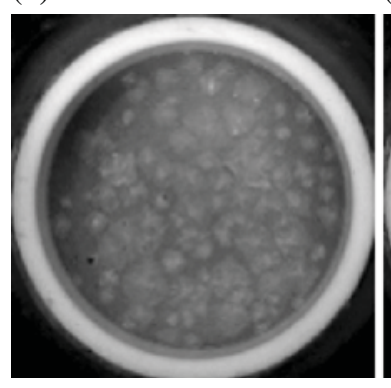

(b)

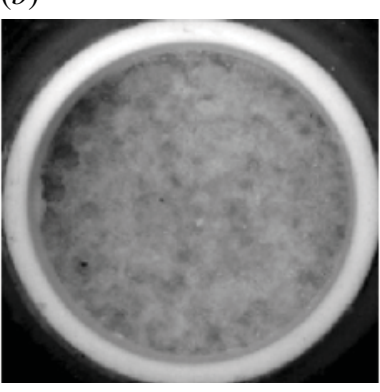

(c)

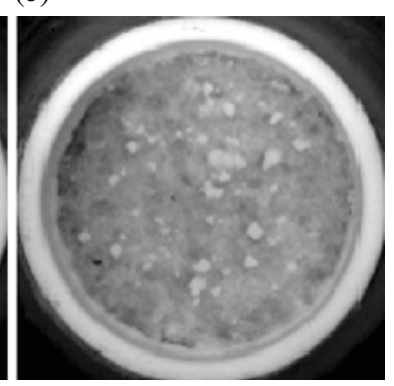

FIgURE 24. Top views of efflorescence during $(a)$ phase $1,(b)$ phase 2 and $(c)$ phase 3 for the beads $(50-62 \mu \mathrm{m})$.

comparable with that in the first phase. An explanation can be proposed from the images of the efflorescence shown in figure 24.

Over a significant period of phase 1 the efflorescence is patchy (figure 24a) and then progressively becomes crusty (figure 24b). As a result of the crust formation, the evaporation rate is much smaller during phase 2. The somewhat unexpected result is that patches form on top of the crust at the end of the second phase (figure $24 c$ ). As a result, the evaporation rate increases up to values comparable with those observed during the first phase. These results confirm that efflorescence patches do not hinder the evaporation whereas a crusty efflorescence can very significantly reduce the evaporation. Also, this result illustrates that a mixed efflorescence, i.e. both crusty and patchy, can form. One possible element of explanation is as follows. As discussed in $\$ 4$, the ion concentration in the porous medium is governed by (4.1). The advection effect induces a concentration peak at the porous medium surface. The greater the Péclet number, the greater the concentration peak. The Péclet is expressed as $P e=\left(J L / A \varepsilon \rho_{\ell} D_{s}\right)$. As a result the ratio of Péclet between phase 1 and phase 2 scales as $P e_{2} / P e_{1} \propto\left(J_{2} / J_{1}\right) \approx 0.35$. As a result of the significant decrease in the advection effect, the concentration in the upper layer of porous medium adjacent to efflorescence should decrease during phase 2. It is surmised that this induces a partial dissolution of the crust opening up or widening passages for the solution through the crust. As a result, new patches form and develop at the top of these passages, which increases the evaporation rate.

\subsection{Patchy-crusty transition from a simple growth model}

Clarifying the mechanisms leading to the patchy-crusty transition when the bead size is varied is among the main open questions in the field. In this respect, it would be certainly interesting to learn more about the internal porous structure of efflorescence as the bead size is varied. Nevertheless, we believe that first insights can be gained from a relatively simple mechanistic model. The main idea is that there is a direct link between the pore size in the efflorescence and the pore size in the porous substrate, that is the bead size in our experiments. The smaller the bead size, the smaller the pore in the efflorescence. Then, since there is a capillary rise in the efflorescence, it is surmised that the viscous resistance associated with the flow of the solution in the efflorescence limits the height of the efflorescence when the pore in the efflorescence becomes sufficiently small. The salt structures would then grow laterally, coalesce and then eventually form a crust. 


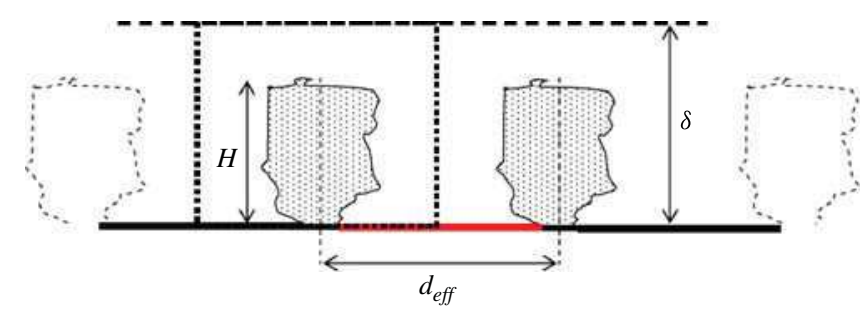

FIGURE 25. (Colour online) Sketch of unit cell of the efflorescence growth model in a two-dimensional lattice.

These ideas, which build on the observation of the films showing the efflorescence development at the surface of the porous medium, can be tested and illustrated from a discrete growth model. Consider the two-dimensional computational domain sketched in figure 25 which is seen as a representative unit cell as (square delimited by the dotted line in figure 25). The domain contains a growing salt structure and a gas region. In the gas region, a pure diffusive transport of the water vapour in the dilute limit is assumed. The growth being slow, the transport of the vapour can be considered as quasi-steady. Under these conditions, the problem to solve in the gas phase reads

$$
\begin{gathered}
\Delta X_{v}=0 \\
X_{v}=X_{v \infty} \quad \text { at } z=\delta \\
X_{v}=X_{v e} \quad \text { at } \Omega_{p m} \cup \Omega_{s w} ; \quad-\rho D \nabla X_{v} \cdot \boldsymbol{n}=0 \quad \text { at } \Omega_{s d},
\end{gathered}
$$

where $\Omega_{s}$ and $\Omega_{p m}$ are the surface of efflorescence and surface of porous medium (free of efflorescence), respectively. Here $\Omega_{s}=\Omega_{s w} \cup \Omega_{s d}$ where $\Omega_{s w}$ and $\Omega_{s d}$ are the sections of the salt structure boundary which are wet and dry, respectively. Spatially periodic boundary conditions are imposed along the vertical edges of computational domain.

From the numerical solution of (6.1)-(6.3), the evaporation flux along the boundary of efflorescence can be computed

$$
j=-\rho D \nabla X_{v} \cdot \boldsymbol{n} \text { at } \Omega_{s} .
$$

The next approximation is to assume that the salt structure locally grows proportionally to the evaporation flux,

$$
j_{c r}=j C_{s a t},
$$

where $j_{c r}$ is the flux of crystallized salt. Thus, the efflorescence cannot grow along the dry section of its boundary.

The salt structure is supposed to be saturated by the solution and a homogeneous isotropic porous medium Combining Darcy's law with the continuity equation leads to the boundary value problem describing the flow in the salt structure. After decomposition of the pressure according to $P=P_{v i s}-\rho_{\ell} g z$, this problem reads

$$
\Delta P_{v i s}=0
$$

with the boundary conditions $P_{v i s}=P_{a t m}$ (atmospheric pressure taken equal to zero for convenience) at $z=0$ (bottom side of computational domain). At the boundary of the 
salt structure, we invoke quasi-steady conditions and express that the evaporation flux balances the liquid flow in the porous medium,

$$
j=-\frac{k_{s}}{\mu_{s}} \nabla P_{v i s} \cdot \boldsymbol{n}
$$

where $k_{s}$ is the permeability of the salt structure. Then we have the compatibility condition that the curvature radius of a meniscus cannot be lower that a certain critical radius $R_{c}$ at any point of the salt structure. The capillary rise compatibility condition thus reads

$$
P_{c t h} \geqslant-\left(P_{v i s}-\rho_{\ell} g z\right),
$$

where $P_{c t h}$ is thus the maximum capillary pressure possible at a meniscus located along the boundary of efflorescence. When this condition is not satisfied, the salt structure cannot grow and the evaporation flux from this cell is then set to zero in the next step of the growth. Actually, the cell is then supposed to become dry (dry efflorescence condition), which means both a zero flux evaporation condition from the considered cell and no salt solution present in the cell. The idea is that such a cell becomes a local barrier to the exchange between the porous medium and the external air owing to salt crystallization.

The above problem is solved on a square lattice of sites. Each site adjacent to the salt structure boundary is a potential growth site. Some randomness is introduced by assuming that the maximum volume that the salt can occupy in a site varies randomly. In this simplified version of the model, the salt structure boundary is formed by a series of edges of the square sites. The efflorescence boundary is therefore a connected series of vertical and horizontal segments. Each segment has the size of the square lattice spacing. The Laplace equations governing $X_{v}$ and $P_{v i s}$ are solved numerically using a standard finite volume technique (Patankar 1980). The growth algorithm can be summarized as follows.

(i) A few connected sites adjacent to the surface of the porous medium (which is located at $z=0$ ) are selected to form the seed of the salt structure. This seed is located in the middle of the bottom edge of computational domain.

(ii) Compute the evaporation rate $J_{i}$ at the boundary of each empty or partially empty cell adjacent to the salt structure. These cells are the boundary cells.

(iii) Compute the pressure field in the salt structure.

(iv) Check for the capillary rise compatibility condition. Set to zero the evaporation rate in the cell where the capillary rise is not possible.

(v) Compute the filling time of the boundary cells as $t_{i}=\rho_{s c} V_{i}(t) /\left(J_{i} C_{s a t}\right)$, where $V_{i}(t)$ is the volume of the cell that can still be 'invaded' by crystallized salt in cell no. i. Here $\rho_{s c}$ is the density of crystallized salt.

(vi) Fully invade the cell corresponding to the minimum time computed in step (iii) and partially invade the other boundary cells: $V_{i}(t+\mathrm{d} t)=V_{i}(t)-\left(J_{i} C_{s a t} \mathrm{~d} t\right) / \rho_{s c}$, where $\mathrm{d} t=\min \left(t_{i}\right)$.

(vii) Go back to step (ii) or stop simulation.

To run the simulation, we have to link the transport properties of the salt structure with the properties of the porous substrate. Thus, we have to specify the permeability $k_{s}$ of the salt structure and the capillary pressure threshold $P_{c t h}$. Since very little is actually known as regards the relationships between the salt structure properties and the porous substrate properties, many options are clearly possible. Here, since the objective is mainly to illustrate the ideas (the characterization of efflorescence microstructure for different porous substrates, using for example 

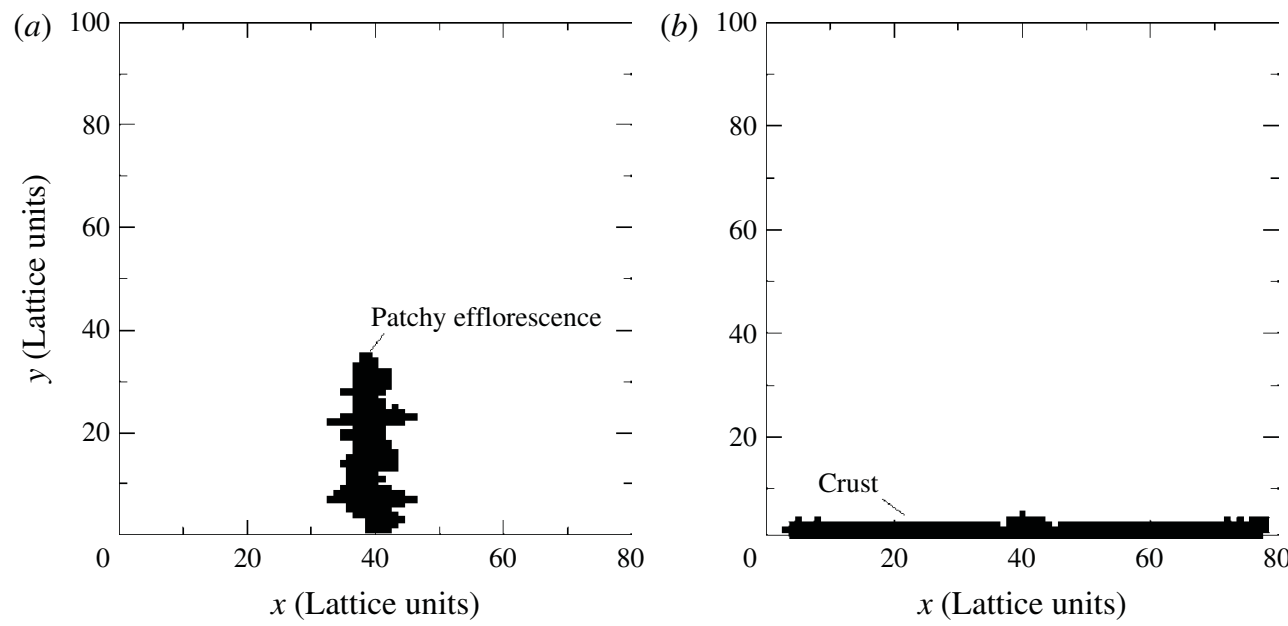

FIGURE 26. Simulation of growth of efflorescence on a lattice: $(a)$ patchy; $(b)$ crusty. The number of invaded cells is the same on both images.

$\mathrm{X}$-ray microtomography techniques is probably possible but represents a considerable work much beyond the scope of the present work), we have chosen for simplicity, but rather arbitrarily, to keep the same capillary pressure threshold (we took $P_{c t h}=6\left(1-\varepsilon_{s}\right) \gamma \cos \theta / \varepsilon_{s} d_{b s}$ with $\varepsilon_{s}=0.1$ and $\left.d_{b s}=100 \mu \mathrm{m}\right)$ regardless of the pore size in the substrate and play with the permeability $k_{s}$, starting from a reference value equal to $k_{s r e f}=\left(\varepsilon_{s}^{3} d_{b s}^{2}\right) /\left(180\left(1-\varepsilon_{s}\right)^{2}\right)$, thus equivalent to the permeability of a packing of beads of size $100 \mu \mathrm{m}$. Since there is indications that a crust is much less permeable that the substrate (Nachshon et al. 2011) whereas patchy efflorescence is clearly not a barrier to transport (Sghaier \& Prat 2009), the idea is to start with a value comparable with the permeability of a substrate for which we do observe patchy efflorescence and then to study the impact of a decreasing permeability.

It should be obvious that other choices, such as varying both the capillary pressure threshold and the permeability would lead to the same qualitative results. The results obtained from this simple growth algorithm using a domain containing $100 \times 80$ cells are summarized in figures 26-28.

When $k_{s}=k_{\text {reff }}$, one obtains a patchy efflorescence as illustrated in figure 26(a). In this case, the capillary rise limit is not reached. The evolution of the evaporation rates reported in figure 27 illustrates the screening effect (evaporation at the surface of the porous medium between efflorescence structures decreases dramatically) and the increase of the evaporation rate from the salt structure with the structure growth, as already discussed in Veran-Tissoires et al. (2012a) and in \$5.2. Note also that the net (or total) evaporation rate does not change very much since the increase in the evaporation rate from the efflorescence is compensated by the decrease in the evaporation rate from the surface of the porous medium free from efflorescence. This is pretty much the situation observed in our experiments with large beads. The situation depicted in figures $26(a)$ and 27 , is actually still observed when the permeability $k_{s}$ is reduced by a factor up to $10^{-6}$ (for the same number of invaded cells as in figure 26(a); for taller efflorescence structures the reduction in permeability corresponding to the occurrence of the capillary rise limit is of course less). For still lower permeabilities, the flow viscous resistance limits the maximum height of the 


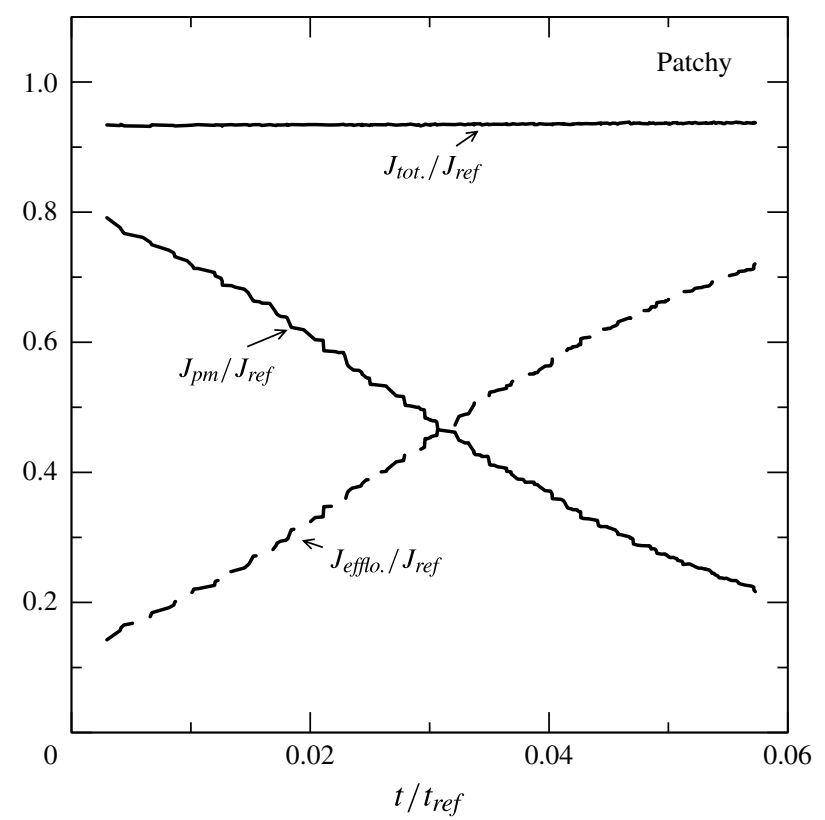

FIGURE 27. Variation of evaporation rates as a function of efflorescence development for the efflorescence pattern shown in figure $26(a)$; $t_{\text {ref }}$ is the time at the end of simulation. Here $J_{\text {efflo }}$ is the evaporation rate from the efflorescence, $J_{p m}$ is the evaporation rate from the porous medium surface free of efflorescence (bottom side of lattice free of efflorescence in figure $26 a$ ); $J_{\text {tot. }}=J_{\text {efflo. }}+J_{p m}$ is the total evaporation rate. The reference evaporation rate $J_{\text {ref }}$ is the evaporation rate in the absence of efflorescence.

efflorescence (capillary rise limit). The results obtained for $k_{s}=10^{-8} k_{\text {reff }}$ are depicted in figures $26(b)$ and 28 . As can be seen, one obtains the formation of a crust covering progressively all of the porous surface (note that the total number of invaded cells is the same in figure $26 a, b$ ).

As shown in figure 28 , the evaporation rate from the crust is very low whereas the total evaporation rate decreases markedly with the development of efflorescence crust. In fact here, the total evaporation rate is essentially equal to the evaporation rate from the porous medium surface free of efflorescence: $J_{t o t} \approx\left(A_{p m}-A_{\text {eff }}\right) / A_{p m} J_{0}$ where $A_{p m}$ and $A_{\text {eff }}$ are the surface of porous medium and the projected surface of efflorescence respectively (thus, $A_{p m}-A_{\text {eff }}$ is the surface of the porous medium free of efflorescence) and $J_{0}$ is the evaporation rate at the beginning of efflorescence formation. The results reported in figure 28 are in qualitative agreement with the experimental result reported in figure 23 for the beads $(5-50 \mu \mathrm{m})$, which shows a severe reduction in the evaporation rate.

\subsection{Discussion}

The experiment for the beads $(5-50 \mu \mathrm{m})$ shows that the formation of the crust severely reduces the evaporation rate (figure 23). Since the evaporation is reduced but not blocked completely, one can assume that there is still a connected network of pores through the crust. Thus, we have to explain the evaporation flux reduction.

The very low permeability leading to the formation of the crust in our model suggests that hygroscopic effects should be included in the analysis. Using the 


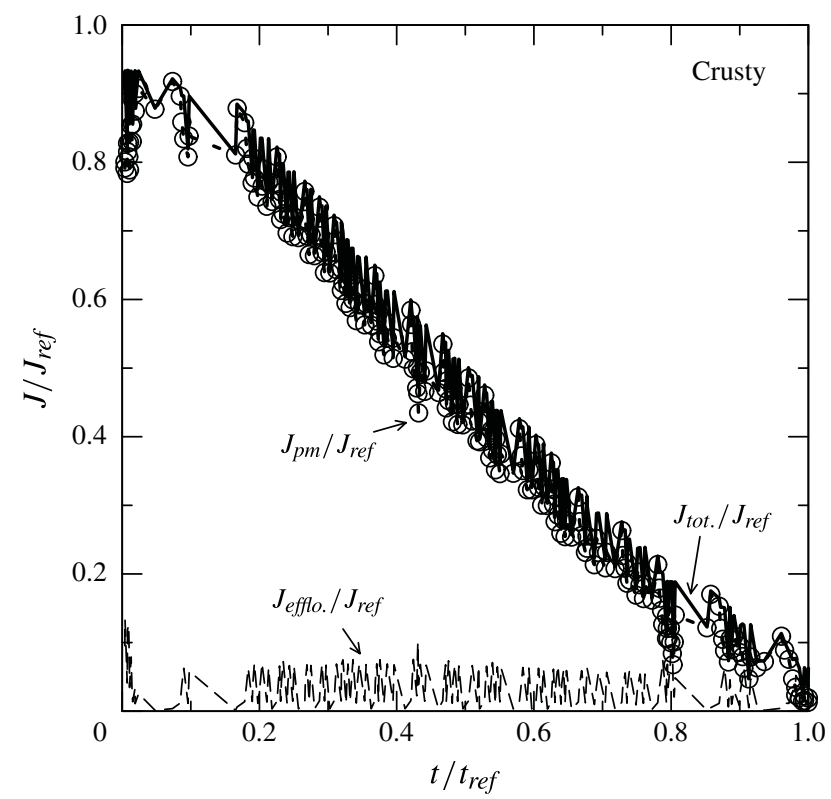

FIGURE 28. Variation of evaporation rates as a function of efflorescence development for the pattern shown in figure $26(b) ; t_{\text {ref }}$ is the time at the end of simulation. Here $J_{\text {efflo. }}$ is the evaporation rate from the efflorescence, $J_{p m}$ (dotted line with empty circles) is the evaporation rate from the porous medium surface free of efflorescence (bottom side of lattice free of efflorescence in figure $26 b$ ); $J_{\text {tot. }}$ (thick solid line) is the total evaporation rate, $J_{\text {tot. }}=J_{\text {efflo. }}+J_{p m}$ The reference evaporation rate $J_{r e f}$ is the evaporation rate in the absence of efflorescence.

Carman-Kozeny relationship $k_{s}=\varepsilon_{s}^{3} d_{b s}^{2} / 180\left(1-\varepsilon_{s}\right)^{2} k_{s}=10^{-8} k_{\text {reff }}$ leads indeed to equivalent bead sizes of the order of $10 \mathrm{~nm}$, which thus would correspond to very tiny pores in the crust). It is well known that the activity of water is reduced at the surface of curved meniscus. The effect is referred to as the Kelvin's effect, which can be expressed as $a_{w}=P_{v s} / P_{v \infty}=\exp \left(-\left(M_{v} / R T\right)(2 \gamma \cos \theta / r)\right)$, where $M_{v}, R$ and $T$ are the water's molecular weight, the universal gas constant and the temperature, respectively. Here we have assumed a meniscus of maximum curvature at the entrance of a cylindrical pore of radius $r$. For water, this relationship gives a significant reduction of water activity $a_{w}$ when the pore size $r$ is in the range (1-100 nm). The picture with the Kelvin effect would not be a full blockage but a severe reduction in the evaporation rate since the water activity at the surface of the crust can become comparable to the activity in the surrounding air. In our experiment with the beads $(5-50 \mu \mathrm{m})$, the evaporation rate is decreased by a factor 14 due to the development of the crusty efflorescence (see figure 23). Expressing the evaporation rate as

$$
J=A D \frac{M_{v}}{R T}\left(\frac{P_{v s}-P_{v \infty}}{\delta}\right)
$$

the water activity at the surface to obtain a decrease of the evaporation rate by a factor 14 must be

$$
a_{w}=\frac{P_{v s}-P_{v \infty}}{14 P_{v s}}+\frac{P_{v \infty}}{P_{v s}} .
$$


Using representative values of our experiment, this leads to $a_{w} \approx 0.15$, which according to Kelvin's relationship corresponds to pores less than $1 \mathrm{~nm}$. This is of course so if one assumes that the effective evaporative surface with the crust is the same as for the porous medium free of efflorescence $(A$ in (6.9)). If the tiny pores on top of crust are in fact scarcely distributed at the surface (the tessellation pattern reported in figure 22(b) suggests for instance that the evaporation could be only from the rim of the tessellation cells), then the evaporation rate could be reduced by the factor 14 with greater pore sizes at the top of efflorescence. As discussed in $\S 3.1$, it is indeed well known that the evaporation rate from a porous surface is reduced when the mean distance between the open pores at the surface is not small compared with the characteristic length of the external mass transfer ( $\delta$ in our problem); see, e.g., Suzuki \& Maeda (1968) for more details.

Suppose for instance pores of aperture $d_{o}$ with a mean distance between pores $\lambda$ such that $\lambda \gg d_{o}$. Then we can assume that the evaporation rate from each pore is about the same as if the pore was alone at the surface. According to Picknett \& Bexon (1977), the evaporation rate from an individual disk over a flat surface is given by

$$
J_{\text {pore }}=2 D d_{o} \frac{M_{v}}{R T}\left(P_{v s}-P_{v \infty}\right) .
$$

We can then determine the number of pores $n_{p}$ leading to the same total evaporation rate as in the experiment (phase of reduced evaporation due to the salt crust with the $5-50 \mu \mathrm{m}$ beads where the evaporation rate is reduced by a factor of 14),

$$
n_{p}=(J / 14) / J_{\text {pore }} \text {. }
$$

The mean distance between is then simply given by

$$
\lambda \approx 2 \cdot\left(\frac{A}{\pi n_{p}}\right)^{2} .
$$

Computations from the above equations for the data corresponding to the experiment with the $5-50 \mu \mathrm{m}$ beads lead to the results shown in figure 29. Note that the Kelvin's effect has been taken into account in the computations, which explains the non-monotonous variation of the ratio $\lambda / d_{o}$ in figure 29 . For example, for pore openings of $100 \mathrm{~nm}$, the mean distance between pores for obtaining the reduced evaporation rate by the factor 14 would be $264 \mu \mathrm{m}$ and the ratio $\lambda / d_{o}$ would be equal to 2640, thus consistent with the assumption $\lambda \gg d_{o}$. The results reported in figure 29 are thus consistent with the idea that the external boundary of the crust is a porous surface with small open pores far apart from each other.

In summary, the discussion presented here suggests that the crusty-patchy transition is more complex than the simple picture where the efflorescence porous microstructure would be the same regardless of the bead size once rescaled by the bead size. We surmised that the crusty-patchy transition is characterized not only by variation in efflorescence mean pore size but also in the geometry of efflorescence microstructure (roughly less porous for the crusty efflorescence than for the patchy efflorescence). There is of course a need to characterize this transition in much more depth. We simply hope that the model and the discussion presented in this section will be instrumental in the development of more advanced studies.

\section{Conclusion}

The formation and evolution of efflorescence structures at the surface of a porous medium is a dynamic process resulting from the interplay between evaporation, ions transport and precipitation-dissolution processes. 


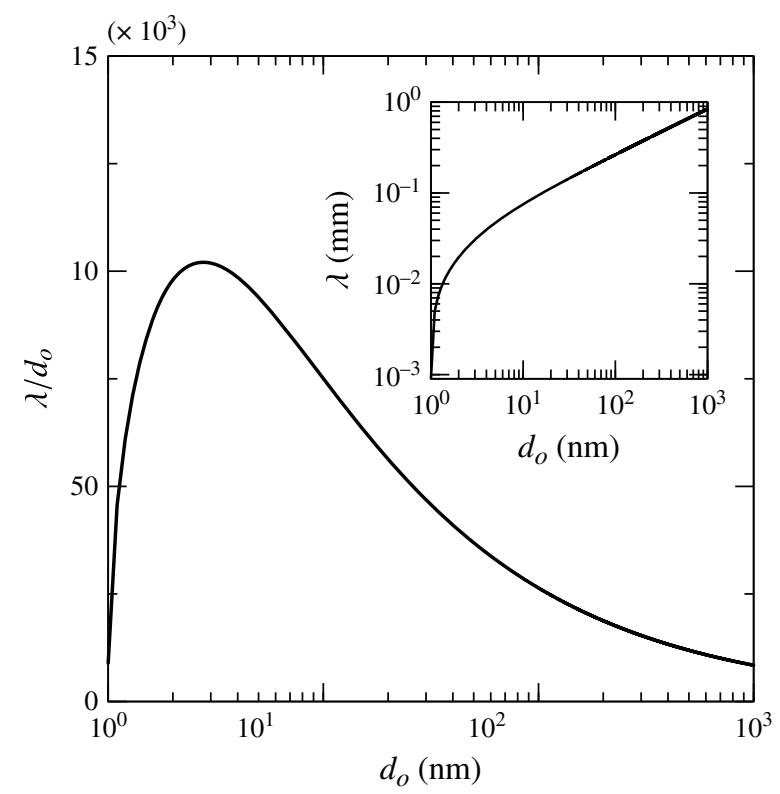

FIGURE 29. Ratio $\lambda / d_{o}$ leading to the same reduced evaporation rate as in the experiment with the $(5-50 \mu \mathrm{m})$ beads (see figure 23) as a function of opening size. Here $\lambda / d_{o}$ is the ratio between the mean distance between openings at the surface and the opening size. The inset shows the mean distance between openings in millimetres.

The incipient precipitation spots at the surface are discrete and must correspond to local maxima in the ions concentration at the surface.

The distribution of the salt concentration peaks at the surface is first impacted by the distribution of evaporation flux at the surface. The greater peripheral flux observed for sufficiently small $\delta$ in our experiments lead to the onset of precipitation at the surface periphery under the form of a sort a fairy ring. This is due to the greater advection effect near the periphery induces there by the greater evaporation fluxes. This effect can be captured by continuum Darcy's scale models. The continuum model is of course unable to predict the discrete nature of the precipitation spots distribution, which was analysed from pore-network simulations.

The discrete distribution of ion concentration maxima or concentration peaks at the surface is intimately related to the disordered nature of porous medium at the pore network scale. Two types of disorder were distinguished: surface disorder and internal disorder. Both disorders contribute to generate velocity fluctuations in the pore space and in turn ion concentration spatial fluctuations owing to the significance of advection in the ion transport. The surface disorder, that is the variation in the surface area of the menisci present at the porous medium surface, is responsible for fluctuations in the local evaporation rates, i.e. in the evaporation rate from the various menisci forming the gas-liquid interface at the porous medium surface. The internal disorder is responsible for fluctuations in ion concentration somewhat similarly as in dispersion problems in homogeneous porous media, that is when a species is transported in a random velocity field.

The surface disorder controls the distribution of ion concentration peaks at the surface at short times whereas the effect of internal disorder becomes dominant when 
the zone of significant concentration gradients adjacent to the surface develops over a distance of a few bead diameters or greater.

The study confirms that supersaturation effects are non-negligible for sodium chloride. The supersaturation effect delays the crystallization and thus has an impact on the localization of concentration peaks at the surface.

Two main types of efflorescence were distinguished: crusty and patchy from both the morphology of efflorescence and the impact of efflorescence development on evaporation rate. The development of patchy efflorescence can increase the evaporation rate whereas the crusty efflorescence significantly reduces the evaporation rate. In the experiments, the transition from patchy to crusty efflorescence was observed in reducing the size of the beads forming the porous medium. This transition was analysed from a simple growth model taking into account the porous structure of efflorescence and assuming that the mean pore size in the efflorescence decreases with the pore size in the underlying porous medium. The analysis suggests that both the viscous resistance to the flow within the efflorescence and the reduction of water activity at the efflorescence external surface, which both increase with decreasing pore sizes contribute to the patchy-crusty transition.

The study is based on several approximations and there are of course many aspects that could be improved. For example, the impact of surface disorder was studied from a model surface, which is clearly much simpler that the surface of a bead packing. It would be interesting to study the evaporation flux distribution from images of the phase distribution at the surface of packing obtained either experimentally using X-ray tomography techniques for example or numerically. This does not seem out of reach.

The patchy-crusty transition certainly deserves further experimental or numerical studies. In particular, it would be interesting to characterize the pore size in efflorescence so as to study the relationship between the pore size in the porous medium and the pore size in the efflorescence for a given evaporation demand. Our growth model of efflorescence suggests that varying the evaporation demand for a given porous medium should also lead to a crusty-patchy (or blocking-non-blocking) transition. This would deserve to be confirmed experimentally.

\section{Appendix}

The appendix gives details on the pore network model. The pressure in the liquid is expressed as

$$
P=P_{h}+\tilde{P}
$$

where $P_{h}=P_{r e s}+\rho_{\ell} g z$ is the hydrostatic pressure distribution in the porous medium; $P_{\text {res }}$ is the pressure at the interface between the porous medium and the bottom reservoir. To determine the pressure field $\tilde{P}$ corresponding to the flow induced by the evaporation process in the porous medium, the mass conservation of the liquid at each liquid node (pore) of network is expressed as

$$
\sum_{i} Q_{i j}=0 \quad i \in\{W, N, E, S, \operatorname{Sup}, \operatorname{Inf}\}
$$

where the volumetric flow rate between two nodes $i$ and $j$ of network is expressed as

$$
Q_{i j}=\frac{g_{i j}}{\mu_{\ell}}\left(\tilde{P}_{i}-\tilde{P}_{j}\right) .
$$


The local hydraulic throat conductance $g_{i j}$ (viscous resistance is neglected in the pores) is expressed as (Poiseuille's law),

$$
g_{i j}=\alpha \frac{r_{l i j}^{4}}{d_{p}},
$$

where $r_{\ell i j}$ is the throat radius. How the numerical factor $\alpha$ is specified is described below.

The boundary condition at the interface between the network and the bottom reservoir is expressed as $P=P_{\text {res }}$ at $z=0$ where $P_{\text {res }}$ is in fact an arbitrary numerical constant. As a result $\tilde{P}=0$ at the network bottom surface. On the sidewall, a zero flow rate condition is imposed ( $g_{i j}=0$ in the corresponding throats). The more interesting boundary condition is the one at the top surface of network. The flow rate in the interfacial throat $n$ at the top surface is expressed as

$$
Q_{n}^{i n t}=\frac{J_{n}(\delta)}{\rho_{\ell}},
$$

where $J_{n}$ is the evaporation rate from the meniscus located at the entrance of the throat. The way of computing $J_{n}$ was discussed in $\S 3.2$. The resulting linear problem is solved numerically using a conjugate gradient method (Saad 1994).

The dissolved salt transport on the network is solved numerically from the following discrete transport equation, which can be seen as a discrete version of a transient convection-diffusion equation,

$$
V_{e} \frac{C_{p}^{t+\mathrm{d} t}-C_{p}^{t}}{\mathrm{~d} t}+\sum_{i \in\{W, N, E, S, \text { Sup }, I n f\}}\left(Q_{i p} \frac{C_{p}^{t+\mathrm{d} t}+C_{i}^{t+\mathrm{d} t}}{2}-g_{D i}\left(C_{i}^{t+\mathrm{d} t}-C_{p}^{t+\mathrm{d} t}\right)\right)=0
$$

where $V_{e}$ is the volume assigned to the element $\left(V_{e}=\right.$ pore volume + half-volume of adjacent throats for an inside pore as shown in figure $14 a$; for the first plane of nodes at the surface see figure 14b); $Q_{i p}$ is the volumetric flow rate in the neighbour throat $i$ (positive when the flow is leaving the pore). The diffusive conductance $g_{D i}$ associated with throat $i$ is expressed as

$$
g_{D i j}=\beta D_{s} \frac{r_{\ell i j}^{2}}{d_{p}},
$$

where $\beta$ is a numerical factor.

The numerical factors $\alpha$ and $\beta$ are specified so that the pore network models lead to the same concentration and velocity fields as the continuum approach when the network is perfectly ordered (all of the throats with the same size; all of the pores with the same size). From the comparisons with the finite difference discretized forms of the continuum equations (see $\S 4.2 .1$ ), one obtains

$$
\begin{gathered}
V_{e}=\varepsilon d_{p}^{3} \\
\alpha \frac{r_{\ell}^{4}}{d_{p}}=K d_{p}=\frac{\varepsilon^{3} d_{p}^{3}}{180(1-\varepsilon)^{2}} \\
\beta D_{s} \frac{r_{\ell}^{2}}{d_{p}}=\varepsilon D_{s}^{*} d_{p}=\varepsilon^{1.4} D_{s} d_{p} .
\end{gathered}
$$


The parameters are determined from experimental data for a random packing of monodisperse glass beads (e.g. Dullien \& Dhawan 1974). The throat radii are distributed randomly in the range $\left[0.09 d_{p}, 0.21 d_{p}\right]$ according to a truncated Gaussian distribution $\left(\bar{r}_{\ell}=0.15 d_{p}, \sigma_{r_{\ell}}=0.02 d_{p}\right)$. With this value for $\bar{r}_{\ell}$ and $\varepsilon=0.363$, one obtains from (A 8)-(A 10) $\alpha=1.29$ and $\beta=10.76$. The volume $V_{e}$ of the elements is also varied randomly according to a truncated Gaussian distribution $\left(V_{e} \in\left[0.3 d_{p}^{3}, 0.426 d_{p}^{3}\right], \bar{V}_{e}=0.363 d_{p}^{3}, \sigma_{V_{e}}=0.02 d_{p}^{3}\right)$. The porosity wall effect is taken into account by increasing the throat and pore sizes near the wall so as to satisfy approximately (3.13).

The initial condition is $C=C_{0}$ in the network. The boundary conditions are analogous to those used for the continuum models (salt mass fraction $C=C_{0}$ at the bottom of network, zero flux condition on the lateral sides). The boundary condition in the interfacial bonds (bonds at the top surface of network) is also a zero flux condition so that the mass balance at a node $n$ located close to the interface (see figure $14 b$ ) reads

$$
V e \frac{C_{n}^{t+\mathrm{d} t}-C_{n}^{t}}{\mathrm{~d} t}+Q_{i n f} \frac{C_{n}^{t+\mathrm{d} t}+C_{i n f}^{t+\mathrm{d} t}}{2}-g_{D \text { inf }}\left(C_{i n f}^{t+\mathrm{d} t}-C_{n}^{t+\mathrm{d} t}\right)=0
$$

and can be also expressed as

$$
V e \frac{C_{n}^{t+\mathrm{d} t}-C_{n}^{t}}{\mathrm{~d} t}-\frac{J_{n}}{\rho_{\ell}} \frac{C_{n}^{t+\mathrm{d} t}+C_{i n f}^{t+\mathrm{d} t}}{2}-\beta D_{s} \frac{r_{n}^{2}}{d_{p}}\left(C_{i n f}^{t+\mathrm{d} t}-C_{n}^{t+\mathrm{d} t}\right)=0
$$

where $J_{n}$ is the evaporation rate at the entrance of the considered interfacial bond. Under quasi-steady condition, it follows from (A 12) that,

$$
C_{n}=C_{i n f}+\frac{J_{n} d_{p}}{\rho_{\ell} \beta D_{s} r_{n}^{2}}\left(\frac{C_{n}+C_{i n f}}{2}\right)=C_{i n f}+\frac{\pi U_{z} d_{p}}{\rho_{\ell} \beta D_{s}}\left(\frac{C_{n}+C_{i n f}}{2}\right)
$$

where $U_{z}$ is the cross-section averaged velocity in the interfacial bond induced by the evaporation process.

\section{REFERENCES}

Bear, J. 1972 Dynamics of Fluids in Porous Media. Dover.

Bird, R. B., Stewart, W. E. \& Lightfoot, E. N. 2002 Transport Phenomena. 2nd edn. John Wiley and Sons.

Blunt, M. J., Jackson, M. D., Piri, M. \& Valvatne, P. H. 2002 Detailed physics, predictive capabilities and macroscopic consequences for pore-network models of multiphase flow. Adv. Water Resour. 25, 1069-1089.

Carey, V. P. 2008 Liquid-Vapor Phase Change Phenomena. 2nd edn. Taylor \& Francis.

Chatterji, S. 2000 A discussion of the paper 'Crystallisation in pores' by G.W. Scherer. Cem. Concr. Res. 30, 669-671.

Coussy, O. 2006 Deformation and stress from in-pore drying-induced crystallization of salt. J. Mech. Phys. Solids 54, 1517-1547.

Deegan, R. D., Bakajin, O., Dupont, T. F., Huber, G., NAgel, S. R. \& Witten, T. A. 1997 Capillary flow as the cause of ring stains from dried liquid drops. Nature 389, 827-829.

Desarnaud, J., Derluyn, H., Carmeliet, J., Bonn, D. \& Shahidzadeh, N. 2014 Metastability limit for the nucleation of $\mathrm{NaCl}$ crystals in confinement. J. Phys. Chem. Lett. 5, 890-895. 
Dullien, F. A. L. \& Dhawan, G. K. 1974 Characterization of pore structure by a combination of quantitative photomicrography and mercury porosimetry. J. Colloid Interface Sci. 47 (2), $337-349$.

Eloukabi, H., Sghaier, N., Ben Nasrallah, S. \& Prat, M. 2013 Experimental study of the effect of sodium chloride on drying of porous media: the crusty-patchy efflorescence transition. Intl J. Heat Mass Transfer 56 (1-2), 80-93.

Espinosa-Marzal, R. M. \& Scherer, G. W. 2010 Advances in understanding damage by crystallization: discrete and continuum approaches. Acc. Chem. Res. 43 (6), 897-905.

Goudie, A. \& Viles, H. 1997 Salt Weathering Hazards. John Wiley \& Sons.

Guglielmini, L., Gontcharov, A., Aldykiewicz, A. J. \& Stone, H. A. 2008 Drying of salt solutions in porous materials: intermediate-time dynamics and efflorescence. Phys. Fluids 20, 077101 .

Gupta, S., Terheiden, K., Pel, L. \& Sawdy, A. 2012 Influence of ferrocyanide inhibitors on the transport and crystallization processes of sodium chloride in porous building materials. Cryst. Growth Des. 12 (8), 3888-3898.

Hidri, F., Sghaier, N., Eloukabi, H., Prat, M. \& Ben Nasrallah, S. 2013 Porous medium coffee ring effect and other factors affecting the first crystallization time of sodium chloride at the surface of a drying porous medium. Phys. Fluids 25, 127101.

Huinink, H. P., Pel, L. \& Michels, M. A. J. 2002 How ions distribute in a drying porous medium: a simple model. Phys. Fluids 14 (4), 1389-1395.

Joekar-Niasar, V., Hassanizadeh, S. M. \& Dahle, H. 2010 Dynamic pore-network modeling of drainage in two-phase flow. J. Fluid Mech. 655, 38-71.

Kim, J. -H., OchoA, A. \& Whitaker, S. 1987 Diffusion in anisotropic porous media. Trans. Porous Med. 2 (4), 327-356.

Koch, D. L. \& Brady, J. F. 1985 Dispersion in fixed beds. J. Fluid Mech. 154, 399-427.

Masoodi, R., Pillai, K. M. \& VARAnasi, P. P. 2007 Darcy's law-based models for liquid absorption in polymer wicks. AIChE J. 53 (11), 2769-2782.

MASMOUdi, W. \& PRAT, M. 1991 Heat and mass transfer between a porous medium and a parallel external flow, application to drying of capillary porous materials. Intl J. Heat Mass Transfer 34 (8), 1975-1989.

Nachshon, U., Weisbrod, N., Dragila, M. I. \& Grader, A. 2011 Combined evaporation and salt precipitation in homogeneous and heterogeneous porous media. Water Resour. Res. 47, W03513.

Noiriel, C., Renard, F., Doan, M. L. \& Gratier, J. P. 2010 Intense fracturing and fracture sealing induced by mineral growth in porous rocks. Chem. Geol. 269 (3-4), 197-209.

Norouzi RAD, M., ShOKRI, N. \& SAHIMI, M. 2013 Pore-scale dynamics of salt precipitation in drying porous media. Phys. Rev. E 88, 032404.

Patankar, S. V. 1980 Numerical Heat Transfer and Fluid Flow. Hemisphere Publ. Co.

Pel, L., Huinink, H. \& Kopinga, K. 2002 Ion transport and crystallization in inorganic building materials as studied by nuclear magnetic resonance. Appl. Phys. Lett. 81, 2893-2895.

Peysson, Y., Bazin, B., Magnier, C., Kohler, E. \& Youssef, S. 2011 Permeability alteration due to salt precipitation driven by drying in the context of $\mathrm{CO}_{2}$ injection. Energy Procedia 4, 4387-4394.

Picknett, R. G. \& BeXon, R. 1977 The evaporation of sessile or pendant drops in still air. J. Colloid Interface Sci. 61, 336-350.

Puyate, Y. T. \& Lawrence, C. J. 1998 Wick action at moderate Péclet number. Phys. Fluids 10 (8), 2114-2116.

Puyate, Y. T. \& LAWREnCE, C. J. 1999 Effect of solute parameters on wick action in concrete. Chem. Engng Sci. 54, 4257-4265.

Puyate, Y. T., Lawrence, C. J., Buenfeld, N. R. \& Mcloughlin, I. M. 1998 Chloride transport models for wick action in concrete at large Péclet number. Phys. Fluids 10 (3), $566-575$.

Robinson, R. A. 1945 The vapour pressures of solutions of potassium chloride and sodium chloride. Trans. R. Soc. New Zealand 75 (2), 203-217. 
Rodriguez-Navarro, C. \& Doehne, E. 1999 Salt weathering: influence of evaporation rate, supersaturation and crystallisation pattern. Earth Surf. Process. Landf. 24, 191-209.

SAAD, Y. 1994 SPARSKIT: a basic toolkit for sparse matrix computation. http://www-users.cs.umn.edu/ saad/software/SPARSKIT/paper.ps.

Scherer, G. W. 2004 Stress from crystallization of salt. Cem. Concr. Res. 34, 1613-1624.

Schiro, M., Ruiz-Agudo, E. \& Rodriguez-Navarro, C. 2012 Damage mechanisms of porous materials due to in-pore salt crystallization. Phys. Rev. Lett. 109, 265503.

Sghaier, N. \& Prat, M. 2009 Effect of efflorescence formation on drying kinetics of porous media. Trans. Porous Med. 80 (3), 441-454.

Sghaier, N., Prat, M. \& Ben NAsrallah, S. 2006 On the influence of sodium chloride concentration on equilibrium contact angle. Chem. Engng J. 122, 47-53.

Sghaier, N., Prat, M. \& Ben Nasrallah, S. 2007 On ions transport during drying in a porous medium. Trans. Porous Med. 62, 243-274.

Shahidzadeh, N. \& Desarnaud, J. 2012 Damage in porous media: role of the kinetics of salt (re)crystallization. Eur. Phys. J. Appl. Phys. 60 (2), 24205.

Suzuki, M. \& MAedA, S. 1968 On the mechanism of drying of granulars beds. J. Chem. Engng Japan 1 (1), 26-31.

Svendsen, J. B. 2003 Parabolic halite dunes on the Salar de Uyuni, Bolivia. Sedim. Geol. 155, $147-156$

Theoulakis, P. \& Moropoulou, A. 1999 Salt crystal growth as weathering mechanism of porous stone on historicmasonry. J. Porous Mater. 6, 345-358.

VAfAI, K. 1984 Convective flowand heat transfer in variable-porositymedia. J. Fluid Mech. 147, 233-259.

VERAN-TISSOIRES, S. 2011 Sur le phénomène de cristallisation discrète à la surface ou à l'intérieur d'un milieu poreux. PhD thesis (in French), University of Toulouse.

Veran-Tissoires, S., Geoffroy, S., Marcoux, M. \& Prat, M. 2013 Wicking in Porous Materials: Traditional and Modern Modeling Approaches (ed. R. Masoodi \& K. M. Pillai), chap. 8, Taylor and Francis.

Veran-Tissoires, S., Marcoux, M. \& Prat, M. $2012 a$ Discrete salt crystallization at the surface of a porous medium. Phys. Rev. Lett. 108, 054502.

Veran-Tissoires, S., Marcoux, M. \& Prat, M. $2012 b$ Salt crystallization at the surface of a heterogeneous porous medium. Europhys. Lett. 98, 34005. 